\title{
Actividades Experimentales con Tecnologías en Escenarios de Modelización Matemática
}

\section{Experimental Activities with Technologies in Mathematical Modeling Scenarios}

\author{
Mónica E. Villarreal * \\ ORCID iD 0000-0002-9029-4887 \\ María Mina** \\ ORCID iD 0000-0003-2158-444X
}

\begin{abstract}
Resumen
Este artículo analiza el desarrollo de actividades experimentales realizadas por grupos de estudiantes de segundo año, de una escuela secundaria en la ciudad de Córdoba (Argentina), en el marco de proyectos de modelización abiertos, acompañados por el libre uso de tecnologías. Los estudiantes desarrollaron estos proyectos en escenarios de modelización creados en sus clases de matemática. Nuestra investigación busca indagar acerca de: 1) el aprendizaje de estudiantes que participan en escenarios de modelización matemática activa con actividades experimentales y tecnologías digitales y 2) el papel que desempeñan las tecnologías digitales en procesos de modelización que involucran actividades experimentales. En términos teóricos, se adopta la perspectiva del aprendizaje situado y la visión epistemológica involucrada en el constructo humanos-con-medios. Los aprendizajes se evidencian en la participación de los estudiantes en distintas fases de procesos de modelización matemática, desarrollados en comunidades locales de práctica en contexto educativo. Las tecnologías digitales más empleadas por los estudiantes fueron celulares y cámaras fotográficas, para registrar experimentos y tomar mediciones, y software matemático para representar gráficos y comunicar resultados. Estos usos estuvieron relacionados con las fases de experimentación y resolución. El análisis en profundidad de un proyecto particular muestra la potencialidad y el uso creativo de diversas tecnologías digitales en estas fases. Se destaca que el estudio brinda un ejemplo que responde a recomendaciones curriculares en torno a la inclusión de la modelización y el uso de tecnologías en clases de matemática. Finalmente, se reflexiona acerca de la importancia, para la formación de profesores, de vivenciar experiencias como la aquí reportada.
\end{abstract}

Palabras clave: Modelización matemática. Tecnologías digitales. Actividades experimentales. Aprendizaje situado. Educación secundaria.

\footnotetext{
* Dra. em Educaçao Matemática por la Universidade Estadual Paulista (UNESP). Investigadora Independiente del Consejo Nacional de Investigaciones Científicas y Técnicas (CONICET). Profesora de la Facultad de Matemática, Astronomía, Física y Computación de la Universidad Nacional de Córdoba (FAMAF-UNC), Córdoba, Argentina. Dirección postal: Medina Allende s/n, Ciudad Universitaria, Córdoba, Argentina, CP 5000. E-mail: mvilla@famaf.unc.edu.ar.

** Mgter. en Procesos Educativos Mediados por Tecnología por el Centro de Estudios Avanzados, Facultad de Ciencias Sociales, Universidad Nacional de Córdoba (CEA-FCS-UNC). Profesora de la Facultad de Matemática, Astronomía, Física y Computación de la Universidad Nacional de Córdoba (FAMAF-UNC), Córdoba, Argentina. Dirección postal: Medina Allende s/n, Ciudad Universitaria, Córdoba, Argentina, CP 5000. E-mail: mdelvmina@gmail.com.
} 


\begin{abstract}
This article analyses the realization of experimental activities carried out by groups of second-year students from a secondary school in Córdoba (Argentina), within the framework of open modelling projects with the free use of technologies. The students developed these projects in modeling scenarios created in their mathematics classes. Our research seeks to investigate: 1) the learning of students participating in active mathematical modeling scenarios with experimental activities and digital technologies and, 2) the role that digital technologies play in modeling processes that involve experimental activities. In theoretical terms, the perspective of situated learning and the epistemological vision embedded in the humans-with-media construct are adopted. Learning is evidenced by the participation of students in different phases of mathematical modeling processes developed in local communities of practice in the educational context. The digital technologies most used by the students were their cell phones and cameras, to record experiments and take measurements, and mathematical software to represent graphs and communicate results. These uses were related to the experimentation and resolution phases. The in-depth analysis of a particular project shows the potential and creative use of various digital technologies in these phases. It is important to note that the study provides an example that responds to curricular recommendations around the inclusion of modeling and the use of technologies in mathematics classes. Finally, we reflect on the importance, for teacher education, of living experiences such as the one reported in this study.
\end{abstract}

Keywords: Mathematical modeling. Digital Technologies. Experimental activities. Situated learning. Secondary school.

\title{
1 Introducción: modelización y tecnologías en nuestro contexto
}

La modelización matemática y el uso de tecnologías en contextos educativos se caracterizan por ser tendencias de gran dinamismo y diversidad dentro del campo de la educación matemática. Ambas presentan importantes avances o discusiones teóricas, pero también ponen en evidencia un cierto desfasaje entre tales avances teóricos, las propuestas pedagógicas que los acompañan y lo que, efectivamente, ocurre en las prácticas de aula.

En contextos educativos, la modelización ofrece la posibilidad de crear escenarios que permiten vincular la matemática con el mundo real y, así, dar nuevos sentidos a la actividad matemática. Según Kaiser (2014), la importancia de la modelización a nivel internacional se ve reflejada en muchos curriculum nacionales. Sin embargo, la autora observa también que, a pesar del consenso que reconoce la relevancia de la modelización, aun no es claro cómo integrarla en procesos de enseñanza y aprendizaje.

En coincidencia con lo observado por Kaiser (2014), podemos señalar que en diferentes diseños curriculares, destinados a la educación secundaria en Argentina, ${ }^{1}$ aparecen recomendaciones de trabajo con actividades de modelización matemática. Por ejemplo, en los aún vigentes Diseños Curriculares para la Educación Secundaria de la Provincia de Córdoba

\footnotetext{
${ }^{1}$ Si bien desde el Ministerio de Educación de la Nación se elaboran lineamientos educativos generales, en el Sistema Educativo Argentino no existen Diseños Curriculares Nacionales. Cada provincia o estado elabora sus propios Documentos Curriculares atendiendo a las particularidades del contexto regional en función de los lineamientos nacionales.
} 
(2011) se sugiere considerar la modelización para resolver problemas tanto externos como internos a la matemática, y propiciar el estudio de límites del modelo matemático para explicar una situación o fenómeno dado.

Tales sugerencias se postulan para que los estudiantes puedan describir, analizar o predecir el fenómeno de la realidad modelado mediante la matemática puesta en juego. Entretanto, las actividades relacionadas con la modelización en clases de matemática de la escuela secundaria de nuestro país son escasas y, por lo general, acotadas a la aplicación de modelos ya estudiados. En términos de Muller y Burkhardt (2007), podemos decir que se trabaja más bien con aplicaciones ilustrativas de contenidos matemáticos estudiados previamente. Raramente se observa en clases de matemática el desarrollo de una modelización activa (MULLER; BURKHARDT, 2007) que permita a los estudiantes la creación de modelos a partir de problemas planteados por ellos y el recorrido de todas las fases de un proceso de modelización, sin una anticipación de los contenidos matemáticos que será necesario poner en juego.

Este tipo de práctica requiere que los estudiantes formulen problemas, busquen información de diferentes fuentes, planifiquen sus acciones, tomen decisiones, elijan métodos, seleccionen recursos (tecnológicos, por ejemplo), combinen y apliquen conocimientos matemáticos que consideren pertinentes y comuniquen resultados. Diremos que un proyecto de modelización que para su desarrollo requiera de estas acciones es un proyecto abierto (BOALER, 1998).

$\mathrm{Si}$, ahora, dirigimos la mirada hacia el uso de tecnologías que se propone en contextos educativos, encontramos un reconocimiento claro de sus potencialidades en documentos curriculares de nuestro país. En particular, en los Diseños Curriculares de la Provincia de Córdoba (2011, p. 37) podemos ver que se hace referencia al "uso reflexivo de recursos tecnológicos", y se propone introducir la tecnología como herramienta para comprender y resolver problemas.

En síntesis, observamos que los citados Diseños Curriculares para la educación secundaria recomiendan tanto el desarrollo de actividades de modelización como el uso de tecnologías en clases de matemática. Si bien la realización de actividades de modelización acompañadas de tecnologías no se menciona explícitamente en los Diseños, esta conjunción se muestra promisoria para dar cuenta de ambas recomendaciones curriculares.

Las consideraciones previas plantean un desafío: ¿cómo promover en el ámbito educativo formal una modelización activa acompañada por el uso de tecnologías? Consideramos fundamental crear escenarios de modelización donde los estudiantes 
desarrollen una diversidad de actividades relacionadas con este proceso. En particular, resulta desafiante la creación de escenarios en los que se promueva la vivencia de un proceso de modelización completo y enmarcar, así, el trabajo de los estudiantes en lo que Muller y Burkhardt (2007) denominan modelización activa.

Para Bassanezi (2012), el proceso de modelización implica una serie de fases, en el cual la inicial es la selección de un tema de interés. Luego de elegir el tema, se formulan problemas o preguntas asociadas con el mismo y se inicia una búsqueda de datos o, quizás, se diseña un experimento para obtenerlos (fase de experimentación). Una fase de abstracción comienza cuando se seleccionan variables y se levantan hipótesis o conjeturas. Al traducir las preguntas o problemas enunciados en lenguaje natural para el lenguaje matemático, se inicia un proceso de resolución con el fin de obtener un modelo matemático. El proceso de aceptación o rechazo de tal modelo es la validación. Si el modelo es rechazado, puede comenzar una fase de modificación y un nuevo ciclo se inicia.

El recorrido de todas las fases del proceso resulta fundamental desde una perspectiva de modelización activa. En este caso, los estudiantes son invitados a desarrollar sus propios proyectos de modelización, iniciando ese proceso con la libre elección de un tema de la realidad que sea de su interés. El desarrollo de proyectos abiertos a partir de intereses de los estudiantes es una actividad poco frecuente en clases de matemática. A lo largo de los últimos doce años, hemos desarrollado diferentes experiencias en aulas de escuelas de educación secundaria que han aportado evidencia empírica sobre la creación de escenarios de modelización abierta, que permitieron poner en juego procesos de modelización activa. Algunas, junto a profesores de educación secundaria (ASINARI; FRASSA, 2017; CRISTANTE et al., 2007; MARGUET et al., 2007; MINA et al., 2007). Otras, junto a futuros profesores de matemática de la Universidad Nacional de Córdoba que desarrollaron sus primeras prácticas docentes en escuelas secundarias que disponían de tecnologías (VILLARREAL; ESTELEY， 2017; BORBA; VILLARREAL; SOARES， 2016; VILLARREAL, 2013).

Entre todas las experiencias que se llevaron adelante en el marco de prácticas docentes de futuros profesores, hubo una, en particular, que llamó nuestra atención porque gran parte de los temas seleccionados por los estudiantes y los problemas planteados condujeron a la necesidad de realizar algún experimento para estudiar un fenómeno físico o químico de interés para ellos. Al hablar de experimento nos referimos a la acción y efecto de experimentar en el sentido que se le atribuye a este término en el marco de las ciencias fisicoquímicas o naturales, esto es, "hacer operaciones destinadas a descubrir, comprobar o 
demostrar determinados fenómenos o principios científicos" (RAE, s/d) ${ }^{2}$. Esto implicó que en la clase de matemática se abriera un espacio donde podrían tener lugar diferentes prácticas científicas (OSBORNE, 2014), poco frecuentes en estas clases.

En el marco de esa experiencia, en este artículo nos interesa detenernos en el análisis de proyectos de modelización de naturaleza experimental desarrollados por estudiantes de escuela secundaria y acompañados por el uso libre de tecnologías digitales. Buscamos responder las siguientes preguntas: (1) ¿Qué aprenden los estudiantes al participar en escenarios de modelización matemática activa con actividades experimentales y tecnologías digitales? y (2) ¿Qué papel desempeñan las tecnologías digitales en procesos de modelización matemática que involucran actividades experimentales?

De acuerdo al reporte de Stillman (2019) sobre el estado del arte de la investigación en modelización en la educación matemática, podemos decir que nuestro estudio se enmarca en una línea de investigación empírica, centrada en la actividad de los estudiantes como modeladores. Al mismo tiempo, según el análisis de Brown e Ikeda (2019) donde anticipan futuras líneas de investigación en torno a la modelización matemática en la educación, nuestro estudio también puede encuadrarse en la línea que estos autores denominan recursos de entornos de enseñanza y aprendizaje ricos-en-tecnología para la modelización.

En la próxima sección realizamos una revisión de la literatura en torno a la temática de nuestro estudio para poder contextualizarlo en el escenario internacional.

\section{Modelización matemática con tecnologías... y actividades experimentales}

Desde hace ya algunas décadas la comunidad internacional de educación matemática investiga acerca de los vínculos entre el uso de tecnologías y el proceso de modelización matemática. El rol de la tecnología en la modelización ha sido analizado por autores como Borba y Villarreal (2005), Galbraith et al. (2007), Greefrath (2011) o Doerr, Ärlebäck y Misfeldt (2017), entre muchos otros.

Borba y Villarreal (2005) indican que el uso de tecnologías en la educación matemática requiere de abordajes pedagógicos sinérgicos con tal presencia e indican a la modelización matemática como uno de tales abordajes, afirmando que en la asociación tecnología-modelización existe una armonía natural.

Galbraith et al. (2007, p. 130) señalan el uso de la tecnología como central para el

\footnotetext{
${ }^{2}$ Definición del Diccionario de la Lengua Española de la Real Academia Española.
} 
propósito de abordar problemas auténticos en la escuela y afirman que la integración de la tecnología con la matemática en procesos de modelización crea "desafíos esenciales acerca de los cuales necesitamos saber mucho más".

Greefrath (2011) reconoce que la tecnología puede jugar un papel central en un proceso de modelización, posibilitando el acceso a ciertos modelos matemáticos que serían inaccesibles sin la presencia de tecnologías digitales. Este autor señala que las tecnologías digitales pueden influenciar cada fase de un proceso de modelización, asumiendo un papel protagónico, y no solo ser un apéndice en un ciclo de modelización.

En concordancia con el trabajo de Greefrath (2011) y Doerr, Ärlebäck y Misfeldt (2017) señalan que los medios computacionales potencian los procesos matemáticos involucrados en actividades de modelización. Estos autores reconocen que las tecnologías son actores importantes en los procesos de modelización.

En un trabajo reciente, Molina-Toro, Rendón-Mesa y Villa-Ochoa (2019) presentan una revisión de la literatura en la que analizan modos en que el uso de tecnologías se articula con el proceso de modelización matemática en diferentes investigaciones del campo de la educación matemática. Por un lado, localizan estudios en los cuales la tecnología es vista como un recurso para el desarrollo del proceso de modelización en cualquiera de sus fases, aunque su influencia en ese proceso es situacional. Por otro lado, encuentran trabajos en los cuales la tecnología es considerada como un reorganizador del proceso de modelización, una herramienta indispensable para dar cuenta de las necesidades que surgen en el proceso.

Numerosos estudios traen evidencia de que el uso de tecnologías favorece los procesos de modelización, ya sea como recurso o como reorganizador. A continuación, presentamos una revisión de la literatura que incluye trabajos desarrollados con estudiantes de educación secundaria (entre 11 y 18 años), nivel educativo en el que desarrollamos nuestra investigación. En particular, destacamos estudios donde la fase de experimentación, dentro del proceso de modelización, ha adquirido un rol protagónico.

Mina et al. (2007), Villarreal, Esteley y Mina (2010) y Borba, Villarreal y Soares (2016) reportan acerca de experiencias de modelización abierta con estudiantes de primer año (12 y 13 años de edad) que libremente seleccionaron temas de interés y formularon problemas relacionados con ellos. Los dos primeros artículos relatan el desarrollo de proyectos de dos grupos de estudiantes. En el primer caso, querían estudiar la relación entre la edad de un niño y la longitud de la raqueta de tenis más conveniente para la edad. En el segundo caso, la relación entre el costo del transporte y el peso de madera transportada en $\mathrm{kg}$, para una cierta distancia. En ambos proyectos, el uso del software Graphmatica les permitió realizar los 
gráficos de las relaciones y mediante el comando de ajuste de curvas accedieron a expresiones analíticas para esos gráficos, que luego analizaron e interpretaron en el contexto de sus problemas. La tecnología les permitió acceder, de manera anticipada, a un contenido matemático no previsto en el curriculum de primer año, que, de otro modo, no hubieran podido abordar en ese momento. Por su parte, el proyecto que se reporta en Borba, Villarreal y Soares (2016) muestra la influencia de la Internet en los tipos de problemas formulados y su importancia como posible fuente de datos.

El uso de datos publicados en Internet - tales como gráficos o fórmulas - que pueden ser utilizados para el desarrollo de proyectos de modelización fue también abordado por Diniz y Borba (2012) que mostraron evidencia de que datos extraídos de Internet parecieran ser tratados por los estudiantes como si fueran empíricos, y también advirtieron acerca de la necesidad de desarrollar en los estudiantes una mirada crítica para la búsqueda, interpretación y lectura de tales datos.

La generación de datos empíricos, a partir del uso de simuladores virtuales, muestra nuevas oportunidades para el uso de tecnologías en diferentes fases del proceso de modelización. Greefrath y Siller (2017) se refieren a las potencialidades de las tecnologías digitales en la modelización y en las simulaciones, y destacan su importancia para la realización de experimentos matemáticos que permitan descubrir relaciones matemáticas en diversas situaciones. Las simulaciones son vistas como una oportunidad para responder preguntas acerca del mundo real y, a veces, son la única manera de abordar ciertos problemas (por ejemplo, un derrame de petróleo en el mar). Las simulaciones pueden utilizarse para recolectar datos a ser usados con variados propósitos, inclusive para obtener información acerca del sistema simulado.

En Carreira (2009) se discute la importancia de manipulativos virtuales como dispositivos que permiten vivenciar una matemática experimental. La autora se refiere a los manipulativos virtuales como elementos esenciales en un nuevo paradigma de la educación matemática: matemática-experimental-con-tecnologías, y defiende tres "designios ineludibles de las tecnologías digitales en la actividad matemática de los jóvenes: manipulación/experimentación, visualización, investigación” (CARREIRA, 2009, p. 55, traducción propia).

En Villarreal (2018) también se discute acerca del abordaje experimental-contecnologías en clases de matemática para estudiantes de entre 11 y 12 años, como un modo de vincular la matemática con las ciencias naturales. Se muestra el uso de un simulador de movimiento pendular que, a modo de un laboratorio virtual, permite experimentar, obtener 
datos, estudiar relaciones entre diferentes variables (por ejemplo, entre la longitud del péndulo y el período), generar y comprobar hipótesis y crear modelos físicos.

El estudio reportado por Molina-Toro, Villa-Ochoa y Suárez Téllez (2018), del cual participaron voluntariamente cuatro estudiantes que cursaban su último grado de escolaridad secundaria (16-18 años), se desarrolló en un ambiente de modelización con tecnología que los autores denominaron de experimentación-con-graficación-y-tecnología, y se centró en el estudio de fenómenos de variación periódica simulados a través del software Modellus. Los autores concluyen que el trabajo colectivo de estudiantes-con-medios, en el ambiente de laboratorio generado por los investigadores, posibilita que la modelización matemática cumpla un rol doble: como un recurso para estudiar matemática y como un objeto para ser estudiado en sí mismo, en cuanto componente constitutivo de la actividad matemática en la escuela.

El desarrollo de actividades de modelización a partir de datos recolectados durante la realización de experimentos reales, de distinta naturaleza, es menos frecuente en clases de matemática de la educación secundaria. Rodríguez y Quiroz (2016, p. 95) destacan el rol de la experimentación en el diseño de una clase de matemática basada en modelización. Las autoras indican que la comprensión de fenómenos físicos se vería favorecida por la realización de experimentos en el aula. Los beneficios de la experimentación en las clases de ciencias son ya conocidos. Sin embargo, en el aula de matemática no es común la realización de experimentos, a pesar de que su práctica sería posible "gracias al gran auge tecnológico que llevó a las matemáticas a convertirse en una disciplina empírica”.

Carreira y Baioa (2018) desarrollan, con estudiantes de noveno grado (14-15 años de edad), un proceso de modelización matemática basado en la realización de tareas experimentales sencillas, a fin de investigar el sentido de credibilidad que los estudiantes atribuyen a tales tareas. El estudio se fundamenta en el reconocimiento de la relevancia de las actividades experimentales para la modelización matemática desde la perspectiva de la educación en STEM $^{3}$.

Carreira y Baioa (2015) buscan comprender los efectos que el trabajo experimental con objetos y artefactos provoca en el desarrollo de ideas y conceptos matemáticos de estudiantes de noveno grado, a través de tareas de modelización. En el caso que reporta el estudio, la tarea propuesta fue establecer las dimensiones convenientes de una escalera en la construcción de una casa. Las autoras enfatizan que la realización de actividades

\footnotetext{
${ }^{3}$ Sigla en inglés para Science, Technology, Engineering, and Mathematics.
} 
experimentales con objetos reales como base para el desarrollo de tareas de modelización matemática permite que los estudiantes: aprendan haciendo mientras manipulan, experimentan, conjeturan y validan; utilizan herramientas matemáticas para estudiar las propiedades de los objetos y desarrollan una mejor comprensión de los modelos matemáticos construidos.

Ortega, Puig y Albarracín (2019) realizaron experimentos de enseñanza y entrevistas con estudiantes de dos clases de grado 11 (16 años) y emplearon múltiples aplicaciones en iPads para modelizar dos conocidos fenómenos físicos: el movimiento de una pelota que rebota y el de un resorte que se estira. Siguiendo una pauta de trabajo establecida por el profesor-investigador, los estudiantes, en pares, realizaron experimentos reales de los fenómenos en estudio, que fueron video-grabados con iPads y, luego, respondieron una serie de preguntas. Los estudiantes manifestaron ciertas dificultades para interpretar y aprovechar los recursos disponibles en los aplicativos durante los procesos de matematización e interpretación de los modelos. Sin embargo, los autores concluyen que "las herramientas tecnológicas pueden ser útiles para exigir a los estudiantes que presten atención a aspectos que normalmente les son dados y que tomen decisiones a las que no están acostumbrados". Esto resulta fundamental para "desarrollar la competencia de modelización en tareas del mundo real con herramientas tecnológicas cuando tales herramientas se utilizan de manera significativa" (ORTEGA; PUIG; ALBARRACÍN, 2019, p. 175-176, traducción propia).

Hasta aquí, hemos reportado estudios que dan cuenta de la existencia de una relación sinérgica entre modelización y tecnologías, mostrando modos de uso de las mismas en procesos de modelización matemática. También hemos encontrado que, en algunos casos, las tecnologías no pudieron ser aprovechadas por los estudiantes en los procesos de modelización. Notamos que los trabajos en los cuales se puso en juego la realización de prácticas experimentales para iniciar el proceso de modelización, abordaron fenómenos o situaciones planteadas por los investigadores o los profesores y, en varios casos estuvieron pautados por instrucciones precisas para la realización de los experimentos.

El desarrollo, en clases de matemática, de actividades experimentales apoyadas por el uso de diversas tecnologías como parte de procesos de modelización merece mayor indagación, al igual que el estudio en torno a proyectos de modelización con temas elegidos por los propios estudiantes. En este artículo, pretendemos contribuir para expandir los estudios realizados en estas direcciones considerando, como lo señalan Borba y Villarreal (2005, p. 76) que "la asociación de la experimentación, la tecnología y la modelización exhibe una resonancia natural”. 


\section{Un marco teórico pedagógico y epistemológico}

La naturaleza de las preguntas de investigación, planteadas en la Introducción, requiere establecer una visión de aprendizaje y una perspectiva epistemológica que se refiera al rol de las tecnologías digitales en escenarios de modelización. La noción de escenario de modelización fue introducida en Esteley (2014) para describir un entorno en el cual se busca que los estudiantes vivencien un proceso de modelización completo y que el profesor guíe el desarrollo de múltiples proyectos sin conocer previamente la matemática que se pondrá en juego para llevarlos adelante. Según la autora:

[un] escenario de modelización se caracteriza por la presencia de un conjunto de espacios, situaciones, circunstancias, materiales, acciones e interacciones que confieren un sentido al proceso y con ello transforma ese conjunto en una experiencia cuyo fin es llevar al aula la modelización como abordaje pedagógico (ESTELEY, 2014, p. 86).

Esta noción está vinculada con el concepto de contexto, en el sentido de Lave (1991). Esta autora, basándose en la naturaleza contingente y emergente de las acciones humanas, representa al contexto conformado por dos componentes a los que denomina terreno y escenario. El terreno incluye los aspectos del contexto que ya existen, previos al planteo de las acciones o experiencias de los individuos, y sobre los que los sujetos no tienen control (condiciones institucionales, recursos materiales disponibles etc.). A su vez, Lave (1991, p. 164, 165) explica que el escenario se enmarca en un terreno y "se concibe como una relación entre las personas en acción y los terrenos en los que actúan”. En este sentido, terreno denota el aspecto fijo del contexto, mientras que escenario, se refiere a lo creado por los sujetos para desarrollar sus actividades en interacción con el terreno e interactuando con otros sujetos. En síntesis, la actividad se "constituye dialécticamente en relación con el escenario".

El escenario es generador de la actividad y, a la vez, es generado por ella. La experiencia vivida transforma el escenario dentro del terreno. Además, cualquier cambio del escenario también transforma la actividad que está teniendo lugar y con ello a los sujetos de la experiencia. Generar un escenario de modelización en un terreno educativo, pone en evidencia espacios, acciones, sujetos, artefactos, experiencias y conocimientos que confieren sentido y particularidad al escenario y con ello a los procesos de aprendizaje vividos en el mismo.

A fin de analizar aprendizajes en escenarios de modelización y en coherencia con el modo en que se define esa última noción, consideramos adecuado adoptar una perspectiva que concibe al aprendizaje, no como un producto, sino como un proceso situado social, cultural e 
históricamente (LAVE; WENGER, 1991). Para Lave y Wenger (1991) el aprendizaje se entiende como un aspecto integral e inseparable de la participación en las prácticas sociales.

Lave (1996, p. 150) afirma:

Dondequiera que las personas se involucren durante períodos sustanciales de tiempo, día a día, en hacer cosas en las que sus actividades en marcha son interdependientes, el aprendizaje es parte de su participación cambiante en prácticas cambiantes (traducción propia).

El término práctica merece especial atención. Para Wenger (2001, p. 71), el “concepto de práctica connota hacer algo, pero no simplemente hacer algo en sí mismo y por sí mismo; es hacer algo en un contexto histórico y social que otorga una estructura y un significado a lo que hacemos". Así, la práctica no puede pensarse en abstracto o de manera aislada.

Wenger (2001, p. 99) asocia la práctica con la formación de comunidades a través de la noción de comunidad de práctica, que es un constructo central en la teoría del aprendizaje situado. Son tres las dimensiones de la relación entre comunidad y práctica que hacen que la práctica se convierta en "la fuente de coherencia de la comunidad": compromiso mutuo, empresa conjunta y repertorio compartido.

El compromiso mutuo se refiere a las relaciones que se establecen entre los participantes de la comunidad por medio de las cuales pueden llevar a cabo la práctica de la comunidad. La empresa conjunta resulta de un proceso de negociación colectiva de los participantes de la comunidad creando relaciones de responsabilidad mutua. El repertorio compartido alude a los variados recursos creados o adoptados por la comunidad para la concreción de su empresa, incluyendo rutinas, herramientas, maneras de hacer, relatos, gestos, conceptos etc. (WENGER, 2001).

La perspectiva del aprendizaje situado no fue generada por Lave y Wenger, pensando en el aprendizaje en ámbitos de educación formal. Su empleo en el estudio del aprendizaje de la matemática escolar ha sido analizado, en detalle, por varios autores del campo de la educación matemática (ver los volúmenes editados por WATSON, 1998; WATSON; WINBOURNE, 2008). Algunos de ellos han realizado adaptaciones que resultan compatibles con las ideas originales de Lave y Wenger, y al mismo tiempo, potentes para el análisis de lo que ocurre en aulas de matemática.

Tal es el caso de Winbourne (2008, p. 81, traducción propia), que afirma que es "útil pensar a la escuela y el aula en términos de múltiples intersecciones de prácticas y trayectorias donde esas prácticas se extienden en el espacio y el tiempo más allá de los límites de la institución". El trabajo de Winbourne y Watson (1998) propone el uso de la denominación comunidad local de práctica para pensar, más específicamente, acerca del aprendizaje (y la 
enseñanza) de la matemática en el contexto escolar.

Estas comunidades pueden ser locales en términos de tiempo y espacio, en términos de la vida de las personas; de las prácticas usuales en la escuela y las aulas; pueden surgir en el transcurso de una clase y luego diluirse o permanecer en el tiempo. Además de las restricciones temporal y local, estas comunidades locales muestran también las tres dimensiones que definen a cualquier comunidad de práctica: compromiso mutuo, empresa conjunta y repertorio compartido.

A fin de analizar el papel de las tecnologías en la práctica de modelización, adoptamos la perspectiva epistemológica propuesta por Borba y Villarreal (2005) que asumen que el conocimiento es siempre producido por colectivos constituidos por actores humanos y no humanos, por colectivos que estos autores denominan sistemas de humanos-con-medios. En esta perspectiva, la cognición es considerada una empresa social y no meramente individual, por eso se emplea la palabra humanos en plural. Además, la cognición incluye medios ${ }^{4}$ con los cuales el conocimiento es producido y esos medios son esenciales, no meramente auxiliares o complementarios: diferentes medios permiten producir diferentes conocimientos; por este motivo el constructo se completa con la expresión -con-medios, donde el uso de guiones, uniendo las palabras, pretende poner en evidencia que el sujeto cognitivo es siempre un colectivo constituido por seres humanos y medios.

Según Borba y Villarreal (2005), los medios, en particular las tecnologías digitales, pueden transformar prácticas de distinta naturaleza (escolares, matemáticas, científicas etc.), volver obsoletos ciertos contenidos, hacer accesibles otros que estaban reservados para cursos superiores y modificar formas de conocer. Así, diferentes tipos de medios pueden reorganizar modos de pensar, producir conocimientos o participar en diferentes prácticas.

Esta reorganización puede implicar cambios o transformaciones en clases de matemática, por ejemplo, en el tipo de problemas que pueden formularse, en la forma de resolverlos o en el modo de comunicar el conocimiento. En particular, la creación de escenarios de modelización en terrenos dotados de tecnologías diversas abre la posibilidad de realizar experimentos en clases de matemática. Esta posibilidad es la que nos interesa indagar en este artículo, considerando dos aspectos centrales: los aprendizajes en términos de participación en las prácticas de una comunidad local y el papel de las tecnologías digitales en

\footnotetext{
${ }^{4}$ Para Borba y Villarreal (2005), medio puede ser la oralidad, la escritura o cualquier tipo de herramienta, dispositivo, equipo, instrumento, artefacto o material que resulta de algún desarrollo tecnológico. Incluye no solo computadoras, software, Internet sino también instrumentos de medición, lápiz, papel, pizarrón etc. Los medios pueden ser parte de lo que Wenger (2001) denomina repertorio compartido.
} 
procesos de modelización matemática con actividades experimentales.

La próxima sección presenta el diseño de la investigación. Se incluyen detalles del contexto educativo, en general, y particularidades del escenario de modelización en el cual se focaliza nuestra investigación. Se enumeran los datos recolectados y los procedimientos analíticos seguidos.

\section{Diseño de la investigación}

\subsection{El contexto educativo: terreno y escenario}

Las actividades que se presentan y analizan en este artículo se desarrollaron en las clases de matemática de dos secciones de segundo año, de una institución de educación secundaria pública, de gestión privada, de la ciudad de Córdoba ${ }^{5}$. La profesora de matemática era la misma para ambas secciones. Involucró a 65 estudiantes de entre 12 y 13 años de edad: 31 en una de las secciones (13 mujeres y 18 varones) y 34 en la otra (11 mujeres y 23 varones). Cada aula estaba equipada con una pizarra digital instalada sobre un pizarrón de tiza y contaba con acceso a Internet a través de WiFi.

Cada estudiante poseía una netbook de uso personal en la cual se habían instalado programas específicos para matemática, tales como GeoGebra y Graphmatica, y programas clásicos de ofimática. La carga horaria semanal de matemática era de cinco clases de $40 \mathrm{~min}$. Los espacios físicos donde se desarrollaron las clases fueron esencialmente el aula y el laboratorio de ciencias, el cual disponía de mesadas de trabajo con acceso a agua, gas y electricidad. El laboratorio contaba, también, con instrumentos de medición tales como balanza digital, cinta métrica, cronómetros, material e instrumental de laboratorio etc. En términos de Lave (1991), este era el terreno en el cual la profesora responsable de las clases de matemática montó su escenario para segundo año.

Esta docente incorporaba, entre sus estrategias de enseñanza, la experimentación con tecnologías, la resolución de problemas con contextos reales y hacía un uso intensivo de todos los dispositivos tecnológicos disponibles en la escuela. Ella había habilitado sus cursos para que futuros profesores de matemática de la Universidad Nacional de Córdoba (UNC)

\footnotetext{
${ }^{5}$ La educación secundaria en Argentina es de carácter obligatorio y consta de seis años divididos en dos ciclos de tres años cada uno: el Ciclo Básico (alumnos de 12 a 14 años aproximadamente) y el Ciclo Orientado (alumnos de 15 a 17 años aproximadamente). Cada escuela ofrece al menos una orientación, por ejemplo: Ciencias Naturales, Ciencias Sociales etc.
} 
realizaran prácticas profesionales docentes. En 2012, dos estudiantes de la UNC realizaron ahí sus prácticas ${ }^{6}$. Cada una de estas estudiantes, que a partir de ahora llamaremos practicantes, estuvo a cargo de una sección de segundo año, aunque la propuesta de enseñanza fue la misma en ambas secciones.

Las prácticas fueron supervisadas por una docente de la universidad y por la profesora a cargo de los cursos $^{7}$. Ambas asesoraron el diseño de la planificación y acompañaron su implementación en aula. La docente del curso propuso a las practicantes: realizar una experiencia de modelización abierta y constituir en objeto de enseñanza y de aprendizaje las fases de un proceso de modelización, contenidos propuestos en su planificación anual. En términos de Lave (1991), el terreno en el cual las practicantes montaron sus escenarios estuvo definido por las condiciones establecidas por la profesora del curso, sumadas a las condiciones escolares antes descriptas.

A fin de dar cuenta de esta propuesta, las practicantes decidieron crear dos escenarios de modelización, donde los estudiantes asumieron un protagonismo diferente. El primero de ellos, con actividades más guiadas y pautadas, estuvo basado en la realización de una actividad experimental, propuesta por las practicantes. Tal actividad tenía como objetivo estudiar la variación de la altura del nivel de agua en recipientes de diferentes formas, en función del volumen de líquido ingresado en los mismos ${ }^{8}$.

La actividad fue realizada en el laboratorio de ciencias de la institución, con distintos recipientes e instrumentos de medición a disposición. Esta actividad demandó dos semanas, permitió a los estudiantes desarrollar un experimento en clase de matemática y vivenciar, de manera guiada, las distintas fases de un proceso de modelización. Como cierre de la actividad, las practicantes constituyeron el proceso de modelización como objeto de enseñanza. Mediante una invitación a la reflexión de los propios estudiantes, se caracterizó y dio nombre a cada una de sus fases y se presentó un esquema que sintetizaba visualmente el proceso seguido.

En el segundo escenario de modelización, de carácter abierto, los estudiantes fueron invitados a desarrollar sus propios proyectos de modelización considerando las fases estudiadas en el escenario anterior y empleando el esquema de modelización visto, como organizador para la realización de los nuevos proyectos. Esta actividad demandó tres

\footnotetext{
${ }^{6}$ Mayores detalles de estas prácticas pueden verse en Coirini y Giannone (2012) y Villarreal (2013).

${ }^{7}$ Ambas docentes son autoras de este artículo.

${ }^{8}$ Cabe aclarar que actividades semejantes a esta han sido propuestas y analizadas en diferentes trabajos. Ver, por ejemplo, Carlson et al. (2002) o el trabajo seminal editado por Swan (1985) y producido por el Shell Centre for Mathematical Education.
} 
semanas. En cada una de las secciones de segundo año, se conformaron siete grupos, compuestos por cuatro o cinco integrantes. Luego, cada grupo eligió libremente un fenómeno, situación o tema de su interés para estudiar, formuló problemas relacionados con la temática elegida y los resolvió, recorriendo las fases del proceso de modelización ya vivenciado en el transcurso de la actividad experimental realizada en el primer escenario de modelización.

Los materiales empleados fueron de diversa naturaleza: tecnologías digitales, instrumentos de medición y dispositivos, todos ellos, seleccionados o construidos por los propios estudiantes. En este escenario, las practicantes solicitaban a los estudiantes la entrega por escrito de lo producido en etapas claves del proceso de modelización (selección de temas, formulación de problemas, diseño de experimentos - de ser necesario -). Estos escritos permitían a las practicantes monitorear el avance de cada proyecto, aportar ideas para su evolución, generar nuevas discusiones, sugerir material de consulta, facilitar elementos para el montaje de experimentos y gestionar sesiones de puesta en común para mostrar el estado de avance de cada proyecto.

La creación de este segundo escenario de modelización permitió a los estudiantes desarrollar una experiencia de modelización abierta, tal como lo había solicitado la docente del curso. Es lo ocurrido en este escenario el foco de nuestra investigación. Es importante dejar claro que el mismo no fue diseñado con fines investigativos, sino didácticos. La mirada investigativa sobrevino a posteriori de su implementación en aula. Tal como se explicita en la Introducción, lo que motivó la realización de un trabajo investigativo fue el hecho que varios grupos de estudiantes se propusieran estudiar fenómenos que implicaban la realización de algún experimento, lo que reconocemos como una práctica poco frecuente y estudiada en clases de matemática de la educación secundaria.

\subsection{Los datos}

En el segundo escenario de modelización descripto, cada grupo de estudiantes participó en el desarrollo de un proyecto de modelización, de modo que se produjeron catorce proyectos en total. Durante el desarrollo de las clases, se recolectaron datos en diversos formatos: filmaciones de clases; producciones de cada uno de los catorce grupos, tales como, reportes escritos, videos y archivos de GeoGebra o Graphmatica relacionados con sus 
proyectos; filmaciones ${ }^{9}$ de las presentaciones orales finales que los grupos realizaron para mostrar sus proyectos al resto de la clase y filmaciones de avances parciales, donde cada grupo explicaba sus decisiones y dificultades en el proceso de modelización en curso. Asimismo, contamos con las notas de observaciones registradas por la profesora de la universidad que supervisaba las prácticas.

\subsection{Análisis de los datos}

A fin de responder nuestras preguntas de investigación, dividimos el análisis en tres etapas. En una primera etapa, realizamos una lectura minuciosa de las producciones de los estudiantes, observamos los videos de sus presentaciones y categorizamos cada uno de los catorce proyectos como siendo de naturaleza experimental o no experimental. Un proyecto era considerado experimental cuando implicaba el diseño y realización de un experimento, para responder la pregunta planteada por los estudiantes. De los catorce proyectos, encontramos que ocho de ellos fueron de naturaleza experimental. Estos ocho proyectos son el foco de nuestro análisis.

Una vez identificados los proyectos de naturaleza experimental, iniciamos una segunda etapa analítica a fin de responder la primera pregunta de investigación: (1) ¿Qué aprenden los estudiantes que participan en escenarios de modelización matemática activa con actividades experimentales y tecnologías digitales? Desde la perspectiva teórica adoptada, responder esta pregunta implica dar cuenta de la participación de los sujetos en las prácticas de una comunidad local de práctica, la cual se define como tal a partir del reconocimiento de tres dimensiones: compromiso mutuo, empresa conjunta y repertorio compartido.

Por su parte, tal como lo señaláramos anteriormente al citar a Wenger (2001), participar en las prácticas significa hacer algo en un contexto que otorga significado a lo que se hace. De modo que resulta importante describir, con densidad, lo que los sujetos hacen, a fin de ofrecer evidencia empírica de su participación en las prácticas de la comunidad local. Así, en nuestro estudio identificaremos qué comunidades de práctica reconocemos y definiremos las tres dimensiones que las caracterizan como tales. Asimismo, daremos cuenta de la participación de los estudiantes en la práctica de modelización como evidencia de

\footnotetext{
${ }^{9}$ Cabe señalar que las filmaciones realizadas tenían como objetivo principal que las practicantes pudieran observar sus propias clases y el modo en que trabajaban los estudiantes. Cuando decidimos realizar la investigación, todo el material producido por los estudiantes, las filmaciones realizadas y el registro de observaciones de la supervisora conformaron el corpus de datos para la investigación.
} 
aprendizaje situado.

En una tercera etapa, determinamos qué tecnologías han sido empleadas en cada proyecto y con qué propósitos de modelización, a fin de dar respuesta a nuestra segunda pregunta de investigación: ¿Qué papel desempeñan las tecnologías digitales en procesos de modelización matemática que involucran actividades experimentales? Desde la perspectiva del constructo humanos-con-medios se procuran evidencias del papel reorganizador de las tecnologías en tales procesos. Finalmente, entre todos los proyectos de naturaleza experimental, seleccionamos uno para analizar en profundidad el papel de las tecnologías en el proceso de modelización y describir en detalle la experiencia vivida por el grupo que lo produjo.

\section{Presentación y análisis de resultados}

En esta sección presentamos los resultados de la investigación y su análisis en torno a las dos preguntas de investigación planteadas. En un primer apartado, nos referimos a lo que lo estudiantes aprenden en términos de su participación en pequeñas comunidades locales de práctica que desarrollan proyectos de modelización abiertos con actividades experimentales y tecnologías digitales. En un segundo apartado, abordamos el uso de tecnologías en esos proyectos de modelización y nos detenemos en el análisis detallado de un proyecto particular que presenta un profuso y creativo uso de tecnologías.

\subsection{Pequeñas comunidades locales de práctica que desarrollan proyectos de modelización abiertos con actividades experimentales}

Según la perspectiva del aprendizaje que hemos adoptado como marco teórico (LAVE; WENGER, 1991), dar cuenta de aprendizajes implica dar cuenta de la participación de los estudiantes en las prácticas de la comunidad o comunidades que integran en la escuela. Es por ello que, en primer lugar, definimos a qué comunidad o comunidades nos referimos y cuáles son las características que nos permiten reconocerlas como pequeñas comunidades locales de práctica (WINBOURNE; WATSON, 1998). Luego, ofrecemos evidencia de la participación de los estudiantes en esas comunidades al trabajar en proyectos de modelización de naturaleza experimental.

La invitación a los estudiantes para desarrollar proyectos de modelización matemática en grupos desencadenó la conformación de múltiples pequeñas comunidades locales de 
práctica, inmersas en la comunidad local de práctica de la clase de matemática que, a su vez, es parte de la comunidad escolar. Podemos decir que la gran práctica que definió a la comunidad local de la clase durante el desarrollo de las prácticas docentes que dieron marco a esta experiencia fue: hacer matemática (aplicada) en el ámbito escolar.

Esta práctica está estrechamente asociada con la práctica científica de modelización que, a su vez, implica una serie de actividades subsidiarias tales como: formular problemas, seleccionar variables, levantar hipótesis, diseñar experimentos etc. Las diferentes participaciones de los estudiantes en la práctica de la modelización, realizada en un contexto escolar que alberga múltiples comunidades locales de práctica, es lo que reconocemos como aprendizaje.

Al observar el trabajo de cada uno de los grupos, reconocemos en ellos las dimensiones que definen a una comunidad local de práctica: empresa conjunta, compromiso mutuo y repertorio compartido (WINBOURNE; WATSON, 1998). A partir de la invitación de las practicantes los grupos se reunieron para elegir un tema de interés y definir problemas asociados con ese tema. Comenzaron a conversar, discutieron, intercambiaron ideas, negociaron, algunos preguntaron: ¿podemos elegir cualquier tema? El que quieran, respondía la practicante. Otros averiguaron: ¿tenemos que hacer un experimento? Si hace falta sí, pero depende de lo que ustedes elijan, decía la practicante.

Finalmente, cada grupo llegó a un primer acuerdo respecto del tema a abordar que luego se compartió con el resto de la clase. Es decir, cada grupo definió, de manera autónoma, una empresa conjunta: involucrarse en el estudio de un fenómeno o situación de su elección y llevar adelante un proyecto de modelización matemática. Esta empresa fue asumida por los integrantes de cada pequeña comunidad y requirió del desarrollo de un trabajo compartido, en el cual cada miembro asumió un compromiso mutuo de participación.

Por último, el repertorio compartido de cada comunidad incluyó rutinas, reglas, palabras, instrumentos, modos de hacer, relatos, gestos, símbolos, acciones o conceptos que la comunidad de práctica del aula ya había producido o adoptado en ocasión de la primera experiencia de modelización realizada previamente, e inclusive antes. Por ejemplo, el uso de tecnologías tales como la Internet, los softwares para graficar, o los instrumentos de medición tales como regla o transportador, eran parte del repertorio compartido de la clase como una comunidad local de práctica que ya existía antes de la llegada de las practicantes.

Asimismo, el esquema de modelización con el que se había cerrado la actividad en el primer escenario de modelización, a través del cual se introdujeron las distintas fases del proceso, fue un instrumento que permitió organizar el trabajo con los nuevos proyectos. 
Además, como se verá más adelante, también hubo dispositivos que fueron creados por cada pequeña comunidad local para dar cuenta de su empresa y que fueron parte del repertorio compartido de la misma.

A fin de dar una idea global de los proyectos experimentales desarrollados y la participación de los estudiantes en la práctica de modelización que se puso en juego en cada una de las ocho pequeñas comunidades locales de práctica, se ha elaborado el Cuadro 1. La primera columna identifica cada proyecto con el nombre del grupo que lo produjo y un código que facilitará referencias posteriores; el número entre paréntesis informa la cantidad de estudiantes que conforma cada pequeña comunidad. Las siguientes columnas presentan sucesivamente el tema y los problemas o preguntas que fueron formuladas; las variables seleccionadas y las hipótesis planteadas por los estudiantes. Con excepción el título que encabeza cada tema o problema, y que aparece subrayado, todos los textos del Cuadro 1 fueron producciones de los estudiantes, que extrajimos de sus informes finales. El cuadro brinda evidencia de tres actividades asociadas con fases del proceso de modelización matemática: formular problemas, seleccionar variables y plantear hipótesis.

\begin{tabular}{|c|c|c|c|}
\hline $\begin{array}{l}\stackrel{0}{0} \\
\stackrel{0}{0} \\
0\end{array}$ & Tema/Problema & Variables & Hipótesis \\
\hline 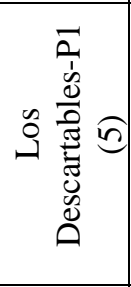 & $\begin{array}{l}\text { Caída de una canica por una } \\
\text { rampa } \\
\text { Explicar la relación entre el } \\
\text { ángulo de inclinación de una } \\
\text { rampa (medida en grados) y el } \\
\text { tiempo (medido en segundos). }\end{array}$ & $\begin{array}{l}\text { Independiente: ángulo } \\
\text { de inclinación de la } \\
\text { rampa. } \\
\text { Dependiente: tiempo en } \\
\text { recorrer la rampa. }\end{array}$ & $\begin{array}{l}\text { La canica va a recorrer la rampa en } \\
\text { menos tiempo mientras la inclinación } \\
\text { de la misma sea más grande. } \\
\text { Si el ángulo es } 0 \text {, la canica no se } \\
\text { moverá. } \\
\text { Creemos que este problema va a ser de } \\
\text { proporcionalidad directa. }\end{array}$ \\
\hline 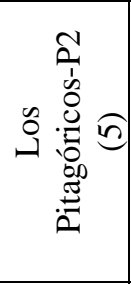 & $\begin{array}{l}\text { Caída de un objeto en un } \\
\text { recipiente con agua } \\
\text { Medir cuánto tarda una goma } \\
\text { de borrar en llegar al fondo de } \\
\text { una probeta, si a ésta se le ha } \\
\text { colocado determinada } \\
\text { cantidad de agua. }\end{array}$ & $\begin{array}{l}\text { Independiente: volumen } \\
\text { de agua en la probeta. } \\
\text { Dependiente: tiempo que } \\
\text { tarda la goma en llegar al } \\
\text { fondo de la probeta. }\end{array}$ & $\begin{array}{l}\text { El tiempo que tarda el objeto en llegar } \\
\text { al fondo del recipiente aumentará } \\
\text { regularmente. } \\
\text { Mientras más agua coloquemos en el } \\
\text { recipiente, más tiempo tardará el objeto } \\
\text { en llegar al fondo. }\end{array}$ \\
\hline 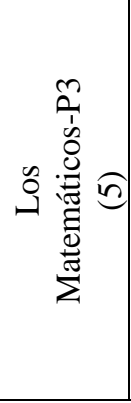 & $\begin{array}{l}\text { Recorrido de un circuito } \\
\text { Saber cuánto tiempo tarda en } \\
\text { recorrer un circuito } \\
\text { determinado, personas con } \\
\text { características diferentes. }\end{array}$ & $\begin{array}{l}\text { Independiente: personas. } \\
\text { Dependiente: tiempo. }\end{array}$ & $\begin{array}{l}\text { Una persona que tiene mayor } \\
\text { entrenamiento tardará menos tiempo en } \\
\text { recorrer el circuito. } \\
\text { Dependiendo de la salud de la persona } \\
\text { tardará mayor o menor tiempo en } \\
\text { recorrer el circuito. } \\
\text { El sexo masculino recorrerá el circuito } \\
\text { en menor tiempo que el sexo femenino } \\
\text { (a menos que el hombre esté en mal } \\
\text { estado con respecto a ella). }\end{array}$ \\
\hline
\end{tabular}




\begin{tabular}{|c|c|c|c|}
\hline 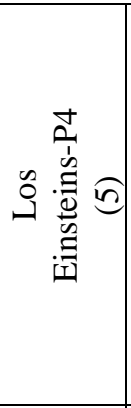 & $\begin{array}{l}\text { Movimiento pendular } \\
\text { Queremos ver si soltando } \\
\text { pelotitas de diferentes pesos } \\
\text { desde distintas alturas, sujetas } \\
\text { por un punto fijo, llegan a } \\
\text { completar una vuelta o ver la } \\
\text { atura máxima a la que llegan. }\end{array}$ & $\begin{array}{l}\text { Independientes: peso de } \\
\text { las pelotitas y altura a la } \\
\text { que se suelta la pelotita. } \\
\text { Dependiente: altura } \\
\text { máxima a la que llega. }\end{array}$ & $\begin{array}{l}\text { La primera idea que surgió fue que una } \\
\text { pelota con mucho peso podía llegar a } \\
\text { dar una vuelta completa. } \\
\text { Luego, nos dimos cuenta de que } \\
\text { ninguna pelota podía llegar a completar } \\
\text { una vuelta, porque necesitaría que se le } \\
\text { aplique cierta fuerza extra, pero que a } \\
\text { mayor altura que tiremos la pelota y } \\
\text { mientras más peso tenga la misma, } \\
\text { llegaría a una altura mayor. }\end{array}$ \\
\hline 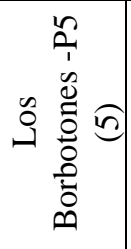 & $\begin{array}{l}\text { Consumo de velas } \\
\text { ¿Cuánto tarda una vela en } \\
\text { apagarse dependiendo del } \\
\text { ancho y de la altura de la } \\
\text { misma? }\end{array}$ & $\begin{array}{l}\text { Independiente: volumen } \\
\text { y forma de la vela. } \\
\text { Dependiente: tiempo. }\end{array}$ & $\begin{array}{l}\text { Mientras más alta sea la vela, más } \\
\text { tiempo tarda en consumirse. } \\
\text { Mientras más ancha sea la vela, más } \\
\text { tiempo tarda en consumirse. } \\
\text { Mientras más ancha y alta sea la vela, } \\
\text { más tiempo tarda en consumirse. }\end{array}$ \\
\hline 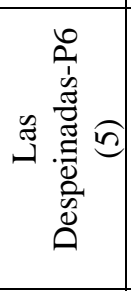 & $\begin{array}{l}\text { Capacidad de absorción de la } \\
\text { vermiculita } \\
\text { ¿Qué cantidad de vermiculita } \\
\text { se necesita para absorber } \\
\text { ciertas cantidades de aceite? }\end{array}$ & $\begin{array}{l}\text { Independiente: volumen } \\
\text { de vermiculita. } \\
\text { Dependiente: volumen } \\
\text { de aceite. }\end{array}$ & $\begin{array}{l}\text { La vermiculita absorbe } 1 \text { vez su } \\
\text { volumen en } \mathrm{cm}^{3} \text {. } \\
\text { Para el doble de aceite se necesita el } \\
\text { doble de vermiculita. } \\
\text { Cuando la capacidad de absorción de la } \\
\text { vermiculita sea la máxima, caerá aceite } \\
\text { por el cuello del embudo. }\end{array}$ \\
\hline 总 & $\begin{array}{l}\text { Derretimiento del hielo } \\
\text { ¿Cuánto tiempo va a tardar en } \\
\text { derretirse un hielo en un vaso } \\
\text { de precipitado con agua a } \\
\text { diferentes temperaturas? }\end{array}$ & $\begin{array}{l}\text { Independiente: } \\
\text { temperatura. } \\
\text { Dependiente: tiempo. }\end{array}$ & $\begin{array}{l}\text { Mientras más caliente esté el agua, más } \\
\text { rápido se derretirá el hielo. } \\
\text { Cuando el hielo se derrita, el agua } \\
\text { bajará de temperatura. }\end{array}$ \\
\hline 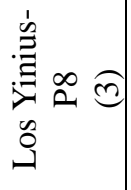 & $\begin{array}{l}\text { Evaporación de alcohol } \\
\text { ¿Qué cantidad de alcohol se va } \\
\text { a evaporar en un determinado } \\
\text { tiempo? }\end{array}$ & $\begin{array}{l}\text { Independiente: tiempo. } \\
\text { Dependiente: altura del } \\
\text { nivel del alcohol. }\end{array}$ & A mayor superficie mayor evaporación. \\
\hline
\end{tabular}

Cuadro 1 - Problemas, variables e hipótesis en los proyectos experimentales de los estudiantes Fuente: elaboración propia

En el Cuadro 1 puede observarse que los temas seleccionados están asociados con fenómenos físicos o químicos, algunos de ellos relacionados con situaciones cotidianas. Si bien las practicantes no sugirieron temas de modelización, las elecciones de los estudiantes parecen surgir en estrecha vinculación con el problema abordado en el primer escenario de modelización, descripto en la Sección 4.1. La naturaleza de los fenómenos elegidos evidencia la significatividad que para los estudiantes tuvo el experimento que se llevó a cabo en el primer escenario de modelización.

La elección de nuevos fenómenos a estudiar, produjo una nueva práctica de modelización caracterizada por el desarrollo de nuevos experimentos, que en esta ocasión deberían ser diseñados por los propios estudiantes. En cada pequeña comunidad, los estudiantes seleccionaron variables adecuadamente y formularon un problema o situación a estudiar en términos de las mismas. Inicialmente, los enunciados de los problemas eran muy 
generales y, con la guía de las practicantes, los estudiantes consiguieron avanzar en la reelaboración de los mismos, introduciendo especificaciones que los tornaban más precisos.

Por ejemplo, la pregunta inicial del grupo Los Pitagóricos (P2), cuyo proyecto refiere a la caída de un objeto en un recipiente con agua, fue: ¿Cuánto tarda un objeto en llegar hasta el fondo de un recipiente al que se le colocó una cantidad de agua determinada y cómo varía cuando repetimos el proceso cinco veces? En la reformulación del problema (ver Cuadro 1) definen qué objeto y qué tipo de recipiente van a utilizar y eliminan la parte final de la pregunta que refiere más bien a un procedimiento experimental.

Respecto de las variables seleccionadas, se destaca que la variable tiempo fue considerada en seis de los ocho proyectos experimentales: P1, P2, P3, P5, P7 y P8. Fue reconocida como variable dependiente en todos los proyectos, salvo en P8. Por lo general, el tiempo era la magnitud que se medía en el experimento y sobre la que se formulaba la pregunta (¿Cuánto tarda una vela en apagarse...? en P5, ¿Cuánto tiempo va a tardar en derretirse un hielo...? en P7). Este hecho hizo que la mayoría de los fenómenos quedasen representados en función de variables independientes diferentes del tiempo, que es una variable usualmente considerada como variable independiente en la física.

Las variables independientes reconocidas en cada proyecto se referían a magnitudes que los estudiantes podían, de alguna manera, variar con cierta facilidad (ángulo de inclinación de la rampa en P1, volumen de agua en una probeta en P2, volumen de vermiculita en P6). Definir el tiempo como variable independiente en un fenómeno físico no era una rutina de esta comunidad local, a diferencia de lo que ocurre en comunidades de práctica conformadas por científicos. Para estos estudiantes, cualquiera de las variables seleccionadas podía ser considerada variable independiente.

El tipo de situaciones elegidas permitió el planteo de hipótesis de diversa índole. Si bien hay algunas basadas en un conocimiento cotidiano del fenómeno a observar (Mientras más caliente esté el agua, más rápido se derretirá el hielo, en P7, o Mientras más alta sea la vela, más tiempo tarda en consumirse, en P5), hay otras que muestran un mayor nivel de elaboración (La vermiculita absorbe 1 vez su volumen en $\mathrm{cm}^{3}$, en P6) e inclusive, en algunos casos, hasta proponen un modelo determinado (Creemos que este problema va a ser de proporcionalidad directa, en P1).

En todos los casos, los experimentos realizados permitieron analizar la validez de las hipótesis planteadas. Analizada la formulación de hipótesis como una actividad asociada a la práctica de modelización, las mismas dan evidencia de la influencia de informaciones localizadas fuera de la comunidad escolar (por ejemplo, en el caso del experimento con 
vermiculita realizado por el grupo P6, una de sus integrantes dijo: Este experimento salió porque la mamá de la Meli para probar la calidad [de la vermiculita] hace un experimento parecido) o dentro de ella (la proporcionalidad directa había sido un modelo estudiado durante el primer escenario de modelización y ya era parte del repertorio compartido de la clase).

El proceso de obtención de datos, visto como una actividad que permitiría responder a las preguntas o inquietudes planteadas, se dio a partir del diseño de diversos experimentos. El diseño de estos experimentos implicó que los miembros de cada pequeña comunidad se comprometieran en la búsqueda de los materiales necesarios para llevarlos adelante o en la tarea de crear dispositivos para poder recolectar datos y estudiar el fenómeno elegido. Tal compromiso fue asumido por los estudiantes que se organizaron y negociaron entre ellos qué elementos debía traer cada uno, discutieron si esos elementos eran apropiados para la recolección de datos del fenómeno considerado, cómo cada elemento se vincularía con los procesos de medición de las variables seleccionadas etc.

Así, cada pequeña comunidad local de práctica se definió en el compromiso mutuo de disponer de lo necesario para la participación en la tarea de desarrollar un experimento. Por su parte, las practicantes como miembros de la comunidad local del aula, realizaron las gestiones necesarias para acceder al laboratorio de ciencias naturales de la escuela, para poder llevar adelante ahí los experimentos y proveyeron algunos de los materiales necesarios para la realización de los mismos cuando fue preciso.

En ciertos casos, el experimento realizado requirió de la creación de algún dispositivo o mecanismo especial para obtener datos (un plano inclinado en P1, un dispositivo pendular en P4, un embudo con papel absorbente en P6); en otros, fue necesario la observación y medición de magnitudes involucradas en fenómenos que requerían de acciones más sencillas (caída de un objeto en el agua en P2, recorrido de un circuito en P3, consumo de velas en P5, derretimiento de un hielo en agua a diferentes temperaturas en P7, evaporación de alcohol en P8). Todas las acciones llevadas adelante en la fase de experimentación activan el repertorio compartido de las comunidades: medir tiempos usando cronómetros, medir ángulos del plano inclinado con el transportador, realizar filmaciones etc.

En síntesis, el Cuadro 1 trae evidencias de que el desarrollo de actividades experimentales realizadas en el marco del escenario de modelización creado, permitió que los estudiantes formularan y reformularan preguntas, seleccionaran variables, levantaran hipótesis, recolectaran datos, actividades propias de la práctica de modelización matemática y de la investigación en ciencias (OSBORNE, 2014). 
Una vez obtenidos los datos, los estudiantes en cada pequeña comunidad local realizaron gráficos empleando softwares específicos (Graphmatica o GeoGebra), que ya eran parte del repertorio compartido de la clase. También contrastaron los datos con las hipótesis inicialmente planteadas, elaboraron conclusiones, escribieron un informe y comunicaron sus resultados a la clase completa. Los momentos de presentación oral de los informes, realizados para el resto de la clase, permitieron observar el nivel de involucramiento de los distintos miembros de cada pequeña comunidad.

La participación de algunos estudiantes daba cuenta de un mayor compromiso mutuo asumido en la empresa compartida. Esos estudiantes podían explicar cada paso realizado en el proceso y responder, de manera pertinente, las preguntas que eran formuladas por compañeros de clase o docentes. Al mismo tiempo, estaban atentos a las intervenciones que les correspondía hacer a los otros miembros de la comunidad y ayudaban con el manejo del cañón de proyección usado para mostrar la presentación. Otros, en cambio, se veían más tímidos en el compromiso con el trabajo y, siguiendo las indicaciones de sus compañeros, trataban de exponer la parte que les tocaba con la mayor claridad posible. Esto revela que los distintos estudiantes que integran una comunidad muestran una participación cambiante en las prácticas cambiantes del aula, y que la empresa compartida pudo lograrse con diferentes niveles de participación en la comunidad de práctica.

La participación de los estudiantes en las diferentes actividades de la práctica de modelización, que fueron desarrolladas por las pequeñas comunidades conformadas en el aula da cuenta de los aprendizajes, en virtud del compromiso asumido por los estudiantes en la empresa de realizar una experiencia de modelización abierta.

\subsection{Las tecnologías digitales en proyectos de modelización de naturaleza experimental}

El Cuadro 2 sintetiza el empleo de distintas tecnologías para el desarrollo de los proyectos de modelización de naturaleza experimental que estamos analizando. La primera columna muestra las diferentes tecnologías elegidas y utilizadas por los estudiantes para el desarrollo de sus proyectos; la segunda enumera los propósitos de uso y la tercera las fases del proceso de modelización relacionadas con esos propósitos. Utilizando el código asignado a cada proyecto, según consta en el Cuadro 1, en la segunda columna se especifica, entre paréntesis, en qué proyectos se puso de manifiesto cada propósito de uso. 


\begin{tabular}{|l|l|l|}
\hline \multicolumn{1}{|c|}{ Tecnología } & \multicolumn{1}{|c|}{ Propósito de uso } & \multicolumn{1}{c|}{$\begin{array}{c}\text { Fase de } \\
\text { modelización } \\
\text { asociada }\end{array}$} \\
\hline $\begin{array}{l}\text { Cámaras o celulares que permiten } \\
\text { registrar imágenes o realizar videos }\end{array}$ & $\begin{array}{l}\text { Realizar mediciones indirectas de tiempo o } \\
\text { distancia (P1-P2-P4) }\end{array}$ & $\begin{array}{l}\text { Experimentación } \\
\text { Software matemático Graphmatica } \\
\text { GeoGebra }\end{array}$ \\
$\begin{array}{l}\text { Representar datos en gráficos cartesianos (P1-P2- } \\
\text { P4-P5-P6-P7) } \\
\text { Generar datos (P4) }\end{array}$ & $\begin{array}{l}\text { Resolución } \\
\text { Experimentación }\end{array}$ \\
\hline Planilla de cálculo & Realizar gráficos de barra (P3) & Resolución \\
\hline Software de edición de videos & Capturar imágenes para generar datos (P4) & Experimentación \\
\hline Cronómetro & Medir tiempos para generar datos (P3-P5) & Experimentación \\
\hline
\end{tabular}

Cuadro 2 - Propósitos de uso para las tecnologías y relación con el proceso de modelización Fuente: elaboración propia

Puede verse, en el Cuadro 2, que las tecnologías más empleadas fueron cámaras o celulares y algún software matemático. En el caso de los dispositivos para registrar imágenes, se observa que en tres proyectos (P1, P2 y P4) se hizo uso de filmaciones como medios indirectos para tomar mediciones de tiempo o distancia. Este uso está relacionado con la fase de experimentación en la cual se busca obtener datos para construir el modelo. Es importante señalar, que estos dispositivos de registro de imágenes no habían sido usados previamente en el aula de matemática. La incorporación de estos recursos es evidencia de un repertorio compartido en otras comunidades de las cuales participan los estudiantes (por ejemplo, las redes sociales) y que son traídos para la comunidad de práctica local de la clase de matemática. Sin el uso de estos recursos hubiera sido imposible para ellos medir, por ejemplo, el tiempo en que una canica recorría una rampa (P1) mediante la observación directa y el uso de un cronómetro de mano.

El uso del software Graphmatica se puso en evidencia en la fase de resolución. Fue el elegido para representar datos que mostraban relaciones entre las variables seleccionadas en cada situación (tiempo necesario para recorrer una rampa vs ángulo de inclinación de la rampa en P1, tiempo que demora un objeto en llegar al fondo de un recipiente con agua vs volumen de agua en el recipiente en P2 etc.). Los estudiantes ya estaban familiarizados con Graphmatica, mientras que el GeoGebra fue utilizado de una manera original en el desarrollo del P4, como se mostrará en la siguiente sección. Ninguno de los grupos arribó a una expresión analítica que representara la situación modelada. En este escenario de modelización, los gráficos y tablas que muestran relaciones entre variables eran reconocidos como parte del repertorio compartido por la comunidad para obtener, representar y comunicar un modelo matemático.

El Cuadro 2 pone en evidencia, también, que la fase del proceso de modelización en la cual el uso de tecnologías fue más frecuente resultó ser la fase de experimentación. Cabe 
destacar que el proyecto en el cual se observa un mayor uso de tecnologías, en general y en la fase de experimentación en particular, fue el desarrollado por Los Einsteins (P4). El mismo puso de manifiesto un uso diferencial y creativo de filmaciones, software de edición de videos y software matemático. Por este motivo, en la próxima sección mostramos y analizamos con detalle el trabajo de este grupo.

Finalmente, cabe señalar que todos los grupos hicieron uso de procesadores de texto para escribir sus informes y de algún soporte visual para realizar sus presentaciones orales. Aquí, los estudiantes utilizaron recursos de comunicación frecuentes en otros espacios curriculares de la escuela. Videos e imágenes fueron producidas por los grupos P1, P4, P6, P7 y P8 para comunicar o explicar a sus compañeros de clase, los procedimientos experimentales seguidos.

\subsubsection{Un ejemplo: el proyecto P4 del grupo Los Einsteins}

En este apartado se describen, de manera densa, las decisiones del grupo Los Einsteins que conformaron una comunidad local de práctica que llevó adelante un experimento particular. Este grupo explicó que para decidir acerca del tema que estudiarían, se inspiraron en un juego mecánico, que denominaban el martillo, y que se encontraba en un parque de diversiones de la ciudad. En la Figura 1 puede verse una imagen de este juego. Inspirados por su funcionamiento, los estudiantes decidieron hacer un experimento para estudiar el movimiento oscilatorio de diferentes cuerpos suspendidos de un hilo sostenido por uno de sus extremos a un punto fijo. Los estudiantes determinaron variables dependientes e independientes y formularon hipótesis que pueden verse en el Cuadro 1. En sus propias palabras plantearon: queremos ver si soltando pelotitas de diferentes pesos desde distintas alturas, sujetos por un punto fijo, llegan a completar una vuelta o ver la altura máxima que llegan. Para ello, construyeron un dispositivo que puede verse en la Figura 2. El mismo consiste de dos soportes verticales y paralelos, apoyados sobre una base plana, que sostienen una varilla en posición horizontal de la cual pende un hilo con una pelotita en el extremo inferior. La longitud del hilo es de $26,5 \mathrm{~cm}$. Este dispositivo pendular tiene adosada una circunferencia graduada de $0^{\circ}$ a $360^{\circ}$, hecha en papel, que permite medir desde qué ángulo se suelta la pelotita. 


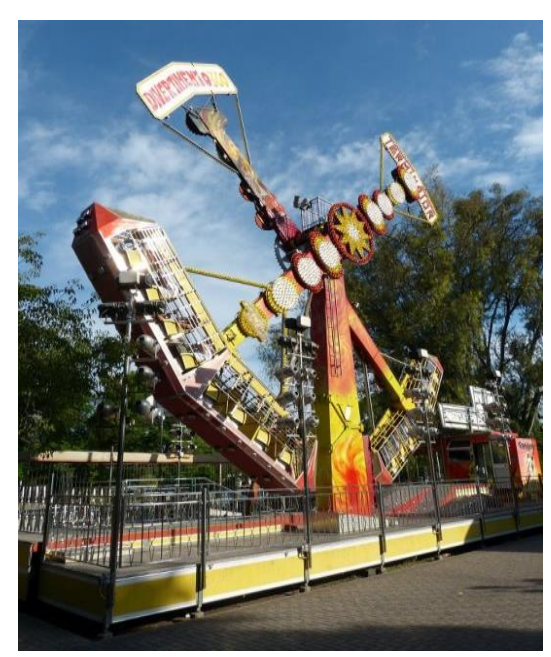

Figura 1 - Juego mecánico El martillo Fuente: Imagen ilustrativa extraída de Internet

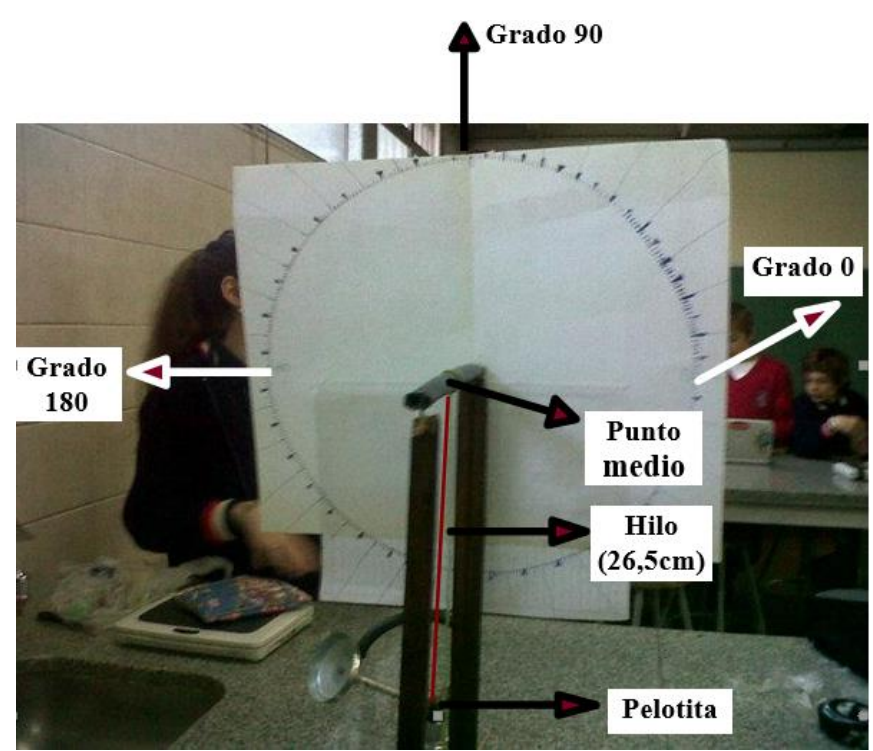

Figura 2 - El dispositivo Fuente: Informe escrito del grupo Los Einsteins

Según las expresiones de los estudiantes durante una exposición sobre el estado de avance de su proyecto, la construcción de este dispositivo implicó distintas dificultades a sortear: ¿cómo suspender las pelotitas al final del hilo y mantener la misma longitud?, ¿qué usar, un hilo o un alambre rígido?, si se usa un hilo, ¿cómo lograr que no se doble en cada tirada?, ¿qué implemento se podría agregar al dispositivo para luego medir alturas?, ¿cómo adosar la circunferencia graduada para que quede perpendicular a la base?

A fin de dar cuenta de sus inquietudes, el grupo diseñó un experimento, usando el dispositivo que había construido. Primeramente, seleccionaron cuatro tipos diferentes de pelotas: una canica (5 gr), una esfera de acero (21,6 gr), una pelota de golf de plástico (15 gr) y una pelota de telgopor (1,2 gr). La disposición de una balanza digital en el laboratorio, que fuera solicitada por los estudiantes, les permitió medir con precisión los pesos de las pelotas.

Luego, cada pelota fue arrojada desde cuatro alturas diferentes, asociadas con los siguientes ángulos: $20^{\circ}, 0^{\circ}, 320^{\circ}$ y $340^{\circ}$, que eran localizados sobre la circunferencia graduada adosada al dispositivo (ver Figura 2). Los estudiantes explicaron: elegimos estos grados, porque no eran ni tan altos, como para que se nos doble el hilo al soltarlo, ni muy bajos, así la cámara captaba bien hasta donde llegaría la pelotita. La cámara que mencionan en esta afirmación, es una cámara filmadora de propiedad de uno de los estudiantes, que ellos mismos decidieron utilizar y que se convirtió en una herramienta fundamental para lograr las mediciones de la altura máxima a la que llegaba cada pelota al ser arrojada desde distintas alturas iniciales.

El Cuadro 3 sintetiza el proceso seguido por los estudiantes para dar cuenta de la 
recolección de datos y su posterior representación gráfica, mostrando las tecnologías utilizadas y las acciones realizadas con cada una.

\begin{tabular}{|c|c|}
\hline Tecnología & Acción realizada \\
\hline Cámara filmadora & Filmación de cada lanzamiento \\
\hline Movie Maker & Reproducción del video en cámara lenta \\
\hline & Detención del video donde la pelota llega al punto máximo \\
\hline & Captura de la imagen \\
\hline GeoGebra & Inserción de la imagen en la vista gráfica de GeoGebra \\
\hline & Realización de una construcción geométrica sobre la imagen insertada en \\
\hline Graphmatica & GeoGebra para obtener la medida de la altura máxima \\
\hline & Representación de los datos en gráficos cartesianos \\
\hline
\end{tabular}

Cuadro 3 - Tecnologías utilizadas por Los Einsteins

Fuente: elaboración propia

Para poder recolectar sus datos, el grupo decidió realizar pequeñas filmaciones para cada una de las cuatro pelotas que eran soltadas desde las cuatro diferentes alturas elegidas. Así, se obtuvieron dieciséis videos. Las decisiones acerca de qué medir y cómo, aparecen condicionadas por las limitaciones de los medios disponibles. Era necesario captar con precisión hasta qué altura llegaba cada pelotita y la cámara daba cuenta de esa necesidad.

Para obtener la medida de la altura máxima a la cual llegaba la pelota arrojada, los alumnos usaron el software denominado Movie Maker para reproducir cada video disminuyendo la velocidad de la reproducción de modo tal que resultara posible detenerla en el momento en que la pelota alcanzaba la máxima altura. Una vez detenida la filmación, capturaban esa imagen y la insertaban en la vista gráfica del GeoGebra. Ya en el GeoGebra, realizaban una construcción geométrica sobre la imagen, tal como se aprecia en la Figura 3, a fin de conseguir la medida de la altura máxima alcanzada por la pelota. Utilizando los comandos de GeoGebra, los estudiantes realizaban el procedimiento que se detalla a continuación.

Primero se marcan los puntos A, B y C. B es el centro de la circunferencia graduada. A y C son puntos sobre la circunferencia. El punto A corresponde a la intersección del hilo con la circunferencia cuando la pelota alcanza la altura máxima en ese lanzamiento. El punto C corresponde al ángulo de $270^{\circ}$. Estos puntos determinan los segmentos $\mathrm{AB}$ y $\mathrm{CB}$. Posteriormente, se traza la recta c, que pasa por C y es perpendicular al segmento CB y luego la recta d que pasa por A y es perpendicular a la recta c. La intersección entre las rectas c y d es el punto D. Una vez determinado el punto D, y haciendo uso de la herramienta Distancia o longitud, se mide la longitud del segmento $\mathrm{AD}$ (1.76, en el caso de la Figura 3) que es la altura máxima alcanzada por la pelota. Finalmente se ubica el punto E, que es el extremo del hilo del cual pende la pelota, y se mide la longitud del segmento EB (4.19, en el caso que muestra la Figura 3) que corresponde a la longitud del hilo que es de 26,5 cm. 
Este procedimiento se repitió para cada pelota (cuatro) y los ángulos elegidos $\left(20^{\circ}, 0^{\circ}\right.$, $340^{\circ}$ y $320^{\circ}$ ), de modo que se obtuvieron dieciséis mediciones. Es interesante notar cómo GeoGebra, siendo una herramienta relativamente nueva para estos estudiantes, ya usada en el estudio de propiedades de figuras geométricas, cambia su función a herramienta de medición indirecta ante la necesidad de medir las alturas alcanzadas por las pelotas. Por otra parte, los estudiantes ponen en evidencia el manejo hábil de las prestaciones de los distintos medios involucrados para realizar mediciones: Movie Maker permite ralentizar la filmación y obtener una imagen estática, y GeoGebra habilita la medición de distancias sobre la imagen (largo del hilo, altura alcanzada por la pelota etc.).

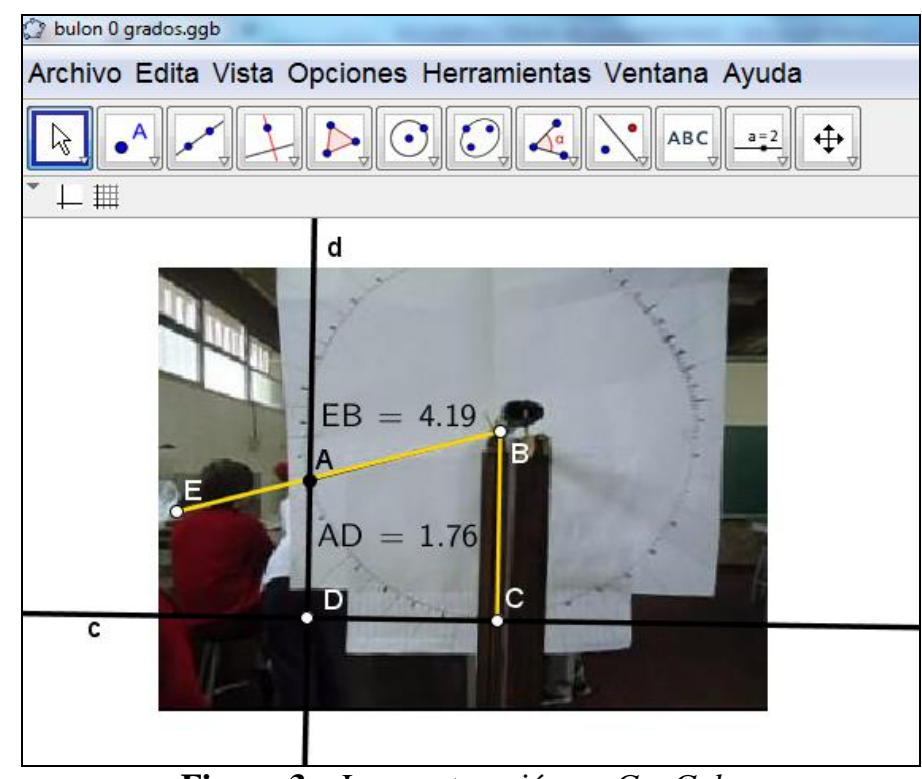

Figura 3 - La construcción en GeoGebra

Fuente: informe escrito del grupo Los Einsteins

Obtenidas las dieciséis mediciones, se observó que la longitud del segmento EB (26,5 $\mathrm{cm}$ ), que representa el hilo que sostiene la pelota difería en las distintas construcciones en GeoGebra. Esto ocurría debido a las unidades de medida del GeoGebra y a la diferencia del tamaño de las imágenes que se insertaban en la vista gráfica del mismo, y sobre las cuales se realizaba la construcción geométrica antes descripta. Este hecho implicó que los valores obtenidos para la medida de $\mathrm{AD}$, que habían convenido en considerar como la altura máxima alcanzada por las diferentes pelotas, no podían compararse y, entonces, decidieron convertir las mediciones a centímetros. Para ello, partiendo del hecho que el segmento EB representaba el hilo y que su longitud en cm era 26,5, para determinar la altura máxima alcanzada por la pelota, en cm, se realizaba la siguiente conversión: $(|\mathrm{AD}| * 26,5) /|\mathrm{EB}|$. Donde $|\mathrm{AD}|$ y $|\mathrm{EB}|$ representan, respectivamente, las longitudes de los segmentos $\mathrm{AD}$ y $\mathrm{EB}$ en unidades de GeoGebra. Una vez realizados los cálculos, los datos finales obtenidos fueron organizados en una tabla que se muestra en la Figura 4. 


\begin{tabular}{|c|c|c|c|c|}
\cline { 2 - 5 } \multicolumn{1}{c|}{} & \multicolumn{4}{|c|}{ Altura máxima a la que llegó la pelota (cm) } \\
\hline $\begin{array}{c}\text { Altura a la que } \\
\text { tiramos la pelota }\left({ }^{\circ}\right)\end{array}$ & $\begin{array}{c}\text { PELOTA 1 } \\
\text { canica } \\
(5 \mathrm{~g} .)\end{array}$ & $\begin{array}{c}\text { PELOTA 2 } \\
\text { esfera de } \\
\text { acero }(21,6 \mathrm{~g})\end{array}$ & $\begin{array}{c}\text { PELOTA 3 } \\
\text { golf } \\
(15 \mathrm{~g} .)\end{array}$ & $\begin{array}{c}\text { PELOTA 4 } \\
\text { telgopor } \\
(1,2 \text { g. })\end{array}$ \\
\hline 20 & 12,7 & 12,94 & 12,51 & 4,53 \\
\hline 0 & 12,59 & 11,13 & 8,02 & 3,67 \\
\hline 340 & 5,82 & 6,47 & 6,36 & 2,95 \\
\hline 320 & 3,44 & 2,55 & 1,89 & 2,12 \\
\hline
\end{tabular}

Figura 4 - La tabla construida por los estudiantes Fuente: informe escrito del grupo Los Einsteins

Las diferencias del tamaño de las imágenes que se insertaban en la vista gráfica de GeoGebra producían medidas que no podían compararse. Para sortear ese obstáculo, los estudiantes debieron recurrir al empleo de una estrategia de conversión que es parte de un repertorio compartido por ellos desde hace años: la regla de tres simple.

Finalmente, para poder trazar un gráfico cartesiano, los estudiantes realizaron un cambio de variable para representar los ángulos seleccionados. Así, en el eje de las abscisas (Altura lanzada), identificaron los ángulos de $20^{\circ}, 0^{\circ}, 340^{\circ}$ y $320^{\circ}$ con los valores $0,20,40$ y 60, respectivamente. La Figura 5 muestra el gráfico obtenido en Graphmatica.

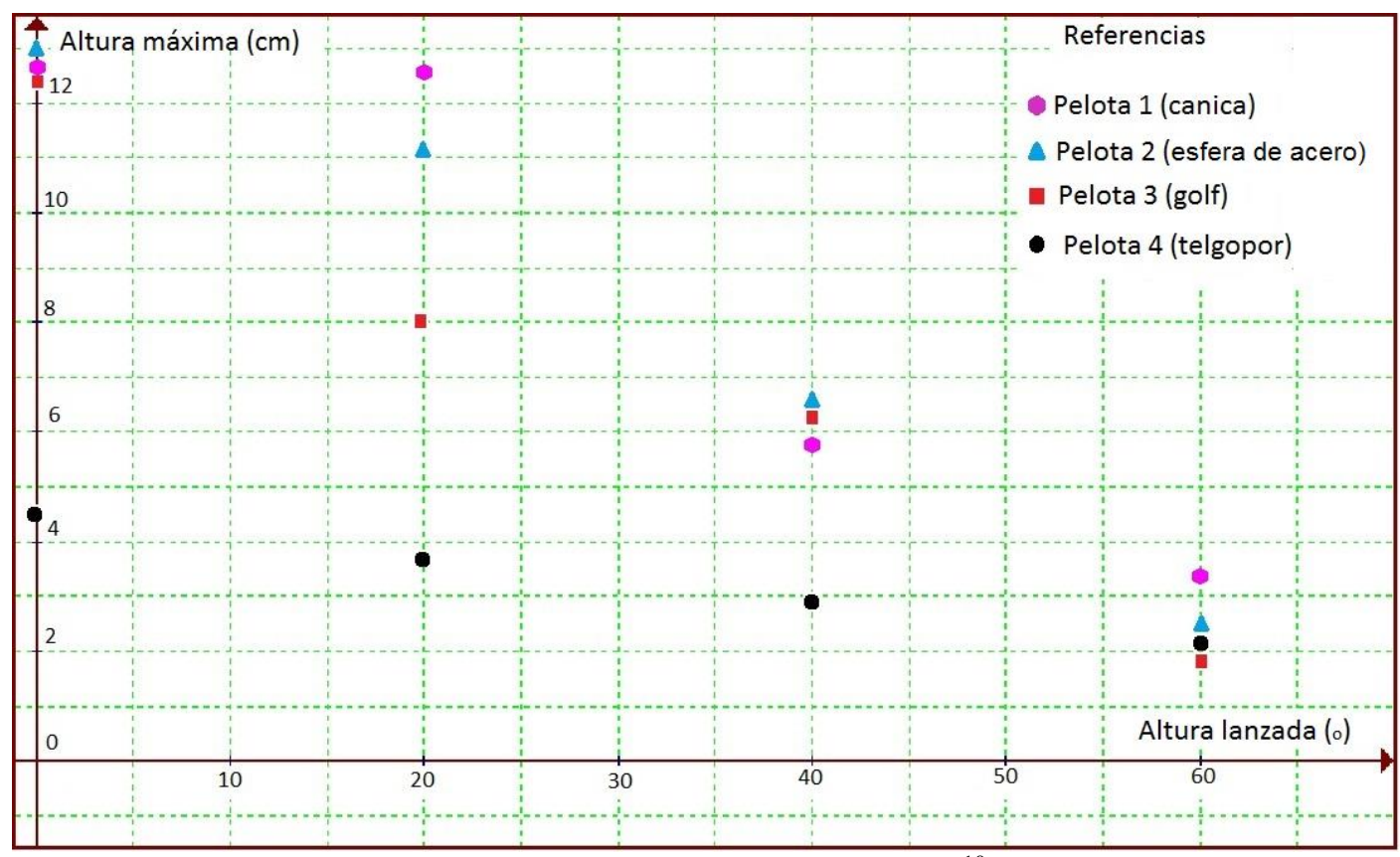

Figura 5 - El gráfico en Graphmatica ${ }^{10}$

Fuente: informe escrito del grupo Los Einsteins

La construcción de la tabla que aparece en la Figura 4 y el gráfico de la Figura 5, son

\footnotetext{
${ }^{10}$ Las referencias que aparecen en el margen superior derecho de la Figura 5 fueron agregadas para facilitar su lectura. Cabe señalar que al revisar las mediciones realizadas por los estudiantes, pudimos constatar errores.
} 
manifestaciones del uso de un repertorio compartido por estos estudiantes para representar simultáneamente múltiples relaciones matemáticas. En el marco del primer escenario de modelización, las practicantes habían trabajado con tablas de dos columnas (una para la variable independiente y otra para la dependiente) y el gráfico cartesiano asociado. En este segundo escenario, Los Einsteins diseñaron una tabla donde la variable dependiente (la altura máxima que alcanza la pelota) toma distintos valores para un mismo valor de la variable independiente (altura a la que tiramos la pelota medido en grados) según el objeto arrojado (canica, esfera de acero etc.).

Para el gráfico cartesiano los estudiantes apelaron a la representación de los pares ordenados de cada relación empleando colores distintos. El nuevo escenario de modelización motivó la realización de un nuevo tipo de representación gráfica que mostraba, simultáneamente, diferentes relaciones y permitía compararlas. De ese modo, una nueva forma de representación era incorporada al repertorio compartido por la comunidad.

Una vez realizado el gráfico, los estudiantes vuelven a sus hipótesis iniciales. Por un lado, verifican que la hipótesis según la cual una pelota con mucho peso podría llegar a dar una vuelta completa no se cumplía. Por otro lado, indican que el experimento les permitió verificar que, a mayor altura en el lanzamiento inicial, mayor era la altura que alcanzaba cada pelota.

Podemos observar que el proceso de modelización seguido por Los Einsteins estuvo fuertemente mediado por tecnologías de diversa naturaleza utilizadas de modos no convencionales: uso del círculo graduado para medir alturas, filmación para registrar experimentos, uso de software de edición de videos para capturar imágenes de la filmación de un experimento y luego realizar mediciones sobre estas, obtención de mediciones indirectas realizadas a partir de construcciones en GeoGebra, representación de todas las situaciones consideradas en un único gráfico cartesiano, entre otros.

Por un lado, fue posible dar evidencia del compromiso mutuo de Los Einsteins en el abordaje de la empresa conjunta de generar un modelo matemático del fenómeno que les interesaba, mediante el repertorio producido por ellos (gráficos, tablas, dispositivos experimentales, procesos originales de medición con tecnologías etc.). Por otro lado, la presentación oral realizada para sus compañeros se constituyó en otra instancia que demostró el compromiso en la participación de estos estudiantes. Esta presentación se caracterizó por una adecuada coreografía de intervenciones negociadas entre sus miembros (qué diría y mostraría cada uno de ellos y en qué momento).

En la siguiente sección, discutimos en torno al aprendizaje y el uso de tecnologías en 
los escenarios de modelización matemática con actividades experimentales. Asimismo, relacionamos nuestro estudio con la bibliografía revisada.

\section{Discusión}

Los proyectos de modelización matemática presentados y analizados fueron producciones de estudiantes que se comprometieron en una empresa de modelización activa (MULLER; BURKHARDT, 2007). En un terreno rico en tecnologías digitales se montó un escenario de modelización en el cual los estudiantes, participando de pequeñas comunidades locales de práctica, fueron capaces de recorrer un proceso de modelización completo, seleccionaron un tema, formularon y reformularon problemas, reconocieron variables pertinentes al problema, establecieron relaciones entre las mismas, levantaron hipótesis, diseñaron e implementaron experimentos, recolectaron datos, verificaron o refutaron hipótesis, construyeron modelos que representaron en tablas y gráficos cartesianos. Tales representaciones, que son parte del repertorio compartido por la comunidad del aula, fueron los objetos que estos estudiantes de corta edad reconocieron como modelos matemáticos y fue lo que las practicantes y docente del curso acordaron como apropiado para ellos, al trabajar con el proceso de modelización como objeto de enseñanza.

La participación de los estudiantes en la práctica de modelización matemática en el contexto escolar tiene un carácter complejo y trasciende el mero aprendizaje de procedimientos y conceptos matemáticos propiamente dichos. Tal como fuera señalado por Boaler (2001), un abordaje de modelización fomenta el desarrollo de una serie de prácticas, además del propio conocimiento matemático, que resultan útiles en situaciones reales. Parafraseando a Lave (1996) y Wenger (2001), el aprendizaje de los estudiantes fue parte de su participación cambiante en prácticas cambiantes. Tales prácticas connotaron hacer algo en un contexto histórico y social: la clase de matemática con una propuesta de modelización activa, la invitación a elegir libremente, en grupos, un tema e investigar sobre él con la matemática y las tecnologías. Esto confirió estructura y significado a lo realizado por cada pequeña comunidad local.

La libertad para seleccionar un tema y los medios a través de los cuales investigarlo, sin poder anticipar el contenido matemático que se pondrá en juego, confiere una particularidad a nuestro estudio ya que existe poca investigación en torno a la modelización abierta en la escuela secundaria. Esta libertad implica, necesariamente, una permanente reconfiguración del escenario de modelización. La necesidad de realizar experimentos 
implicó, por ejemplo, la decisión de trasladarse al laboratorio de ciencias, proveer materiales o traerlos desde casa.

La posibilidad de elegir cualquier tema de la realidad permitió, también, el acercamiento provisorio a contenidos de otras disciplinas, y esto contribuye a la interdisciplinaridad en clases de matemática. En el caso particular del proyecto de Los Einsteins, se abordaron conocimientos de física y procedimientos vinculados con la medición. Decimos que el acercamiento es provisorio ya que, por ejemplo, en el caso de esta pequeña comunidad los alumnos manifestaron la concepción que mientras más peso tuviera la pelota, llegaría a una mayor altura. Esta concepción que surgió en una clase de matemática, podría ser analizada con mayor profundidad junto al profesor de física. Del mismo modo, todo lo referido al efecto y tratamiento de errores de medición podría ser trabajado en clases de ciencias naturales o en las propias clases de matemática.

A pesar de la aparición de concepciones erróneas o la presencia de errores de medición, acordamos con Bassanezi (2012) y Carreira y Baioa (2015) en cuanto a la importancia asignada a la experimentación como un soporte para la comprensión y la validación, reformulación o refutación de hipótesis. Más aún, consideramos que los aportes de nuestro estudio están en línea con lo señalado por Rodríguez y Quiroz (2016), que indican la importancia de la experimentación para la comprensión de fenómenos físicos. Asimismo, coincidimos con la investigación de Carreira y Baioa (2018), quienes destacan la relevancia de actividades experimentales en la modelización como modo de contribuir a la educación en STEM.

En el transcurso de nuestra investigación, observamos el uso coordinado de distintos dispositivos tecnológicos, atendiendo los fines de cada proyecto. Hay evidencias de que todas las pequeñas comunidades apelaron a tecnologías de diferentes maneras, sin embargo, resulta de particular interés para este artículo el caso de Los Einsteins. En el mismo, las tecnologías jugaron un rol esencial y constitutivo del trabajo de los estudiantes, en instancias de crear el dispositivo experimental, recoger datos y tratarlos. Tales usos dan cuenta de la sinergia entre modelización y tecnologías, sinergia que se ve potenciada por la componente experimental que completa esta tríada.

Así, las tecnologías tuvieron roles importantes en las fases de experimentación y resolución trascendiendo el mero uso de software para realizar gráficos cartesianos, escribir un informe o preparar presentaciones de diapositivas que fueron usos comunes detectados en todos los proyectos. Si bien los estudiantes del grupo Los Einsteins lograron dar cuenta de las dificultades para recolectar los datos de su experimento, vale la pena considerar para este fin 
el empleo de aplicativos de iPads como los utilizados en la investigación de Ortega, Puig y Albarracín (2019). Esto hubiera contribuido a que los procesos de medición y recolección de datos fuesen menos complejos. Aunque, quizás en este caso, la tecnología hubiese tenido, según la clasificación de Molina-Toro, Rendón-Mesa y Villa-Ochoa (2019), un rol de recurso para la práctica de modelización.

En el ejemplo analizado, el uso de la tecnología se muestra como constitutivo del propio experimento, en dos sentidos: el registro en video del experimento en sí y, por su intermedio, la obtención de mediciones necesarias para el estudio del fenómeno. Así, podría decirse que el uso de determinada tecnología condiciona el experimento posible de ser realizado y, simultáneamente, la necesidad de obtener datos experimentales condiciona el uso de la tecnología disponible. De este modo, se constata que este proyecto de modelización fue realizado por un colectivo de estudiantes-con-medios (BORBA; VILLARREAL, 2005) que posibilitó un uso creativo de las tecnologías disponibles. En términos de la clasificación de Molina-Toro, Rendón-Mesa y Villa-Ochoa (2019) podemos decir que el rol de la tecnología, en este caso, fue de reorganizador de la práctica de modelización.

\section{Conclusiones}

Lo presentado y discutido en este artículo da cuenta de una experiencia no convencional en clases de matemática donde se integran diversas tecnologías y se trabaja con experimentos asociados a distintos fenómenos. El aspecto no convencional de la práctica desarrollada se vincula, por un lado, con la invitación a los estudiantes a elegir libremente temas para investigar y los modos de hacerlo, lo cual permite la incorporación de nuevos medios, actores, conocimientos y prácticas. Es decir, se pone en evidencia la relación dialéctica entre escenario y actividades, en el sentido de Lave (1991).

Por otro lado, se vincula con una visión de la propia matemática en la que se recupera el valor de las actividades experimentales como medios para pensar matemáticamente. Esta misma visión, también está presente en investigaciones como las realizadas por Molina-Toro, Villa-Ochoa y Suárez Tellez (2018), Greefrath y Siller (2017) y Carreira (2009) en las cuales se utilizan simulaciones o manipulativos virtuales para vivenciar una matemática experimental a través del uso de esos dispositivos.

El escenario de modelización, conformado junto a los estudiantes, posibilitó dar cuenta de las recomendaciones de los documentos curriculares locales en relación a la implementación de actividades de modelización y la incorporación de tecnologías en clases de 
matemática. El estudio brinda evidencias de los aportes de los trabajos con procesos de modelización en clases de matemática como medios para constituir al propio proceso en objeto de enseñanza. Al igual que nuestro estudio, la investigación reportada por MolinaToro, Villa-Ochoa y Suárez Tellez (2018) da cuenta de la importancia de generar escenarios donde el proceso de modelización sea, en sí, objeto de estudio.

Así, tornar explícitas las fases de un proceso de modelización como objeto de enseñanza y aprendizaje permitió, en nuestro caso, sentar las bases para luego estructurar el escenario de modelización abierto. Para los estudiantes contar con un esquema del proceso de modelización se constituyó en un modelo para llevar adelante sus propios proyectos, es decir, este esquema se tornó parte del repertorio compartido de cada pequeña comunidad local de práctica. Estos hechos nos permiten indicar que, con este trabajo, avanzamos en aspectos relativos a la modelización como una componente del curriculum, realizando aportes que pueden resultar de interés para la comunidad internacional de educadores matemáticos, así como para profesores de nuestro país.

El escenario de modelización estudiado es ejemplo de una posible forma para integrar la modelización matemática en los procesos de enseñanza y aprendizaje. El estudio de este escenario ofrece evidencia empírica de los efectos de la integración de la modelización matemática en la práctica escolar. Si bien ameritan un análisis más profundo, tanto el ejemplo de Los Einsteins, como las condiciones teóricas discutidas en torno a la modelización y las tecnologías, se constituyen en aportes que permitirían dar mayor especificidad a lo propuesto en nuestros diseños curriculares, ofreciendo, de esta manera, herramientas teóricas y ejemplos de actividades para el aula a las cuales los docentes de nuestra comunidad podrían apelar.

Si volvemos la mirada hacia factores institucionales y humanos que tienen incidencia sobre el trabajo con modelización matemática o con tecnologías, o con ambas simultáneamente, algunos de ellos resultaron relevantes en la experiencia que presentamos. Referido a lo institucional, debemos reconocer que, si bien la escuela en la que se trabajó ofrece un terrero que dispone de diversas tecnologías a las que los estudiantes pudieron apelar, sin el apoyo de la profesora del curso y los directivos de la institución este trabajo no se podría haber concretado. El apoyo no se refiere solo a lo material o al tiempo que se requiere para llevar adelante este tipo de propuesta didáctica, se refiere también a decidirse a tomar el riesgo de abrir el curriculum escolar de modo tal que los estudiantes se tornen autores de ese curriculum al poder elegir lo que quieren abordar en sus proyectos de modelización, y sostener en el tiempo la decisión de llevar adelante la propuesta con todas sus implicancias. 
Un aspecto que llama a la reflexión es la posibilidad real que tiene un único docente de gestionar una clase en la que se estén desarrollando, en simultáneo, diferentes proyectos de modelización; el trabajo en colaboración con otros colegas parece ser una condición institucional indispensable para realizar este tipo de propuesta. En relación con el factor humano, no podemos dejar de mencionar que tanto la profesora del curso como las practicantes involucradas tuvieron una disposición para innovar en clases, como así también una relación particular con la matemática y una posición epistemológica en torno al uso de tecnologías reconociéndolas como constitutivas del conocimiento que se produce (BORBA; VILLARREAL, 2005). Es decir, la posición asumida por un docente resulta esencial para sostener las decisiones didácticas que se tomen.

Finalmente, cabe señalar que la emergencia de actividades experimentales parece haber sido decisiva para que las tecnologías pudiesen estar presentes en varias fases de los procesos de modelización realizados por los estudiantes. Quizás no cualquier escenario de modelización ofrezca la potencialidad de conjugar, armoniosamente, modelización y tecnologías. Creemos que esta y otras cuestiones levantadas en el artículo ameritan mayor estudio, tal como lo señalan Galbraith et al. (2007): existen desafíos sobre los que necesitamos saber más.

Entre esos desafíos podemos mencionar las decisiones sobre la evaluación en escenarios de modelización que propician el uso de tecnologías. También surgen temas asociados a los modos de contribuir para la formación de docentes o futuros docentes para que puedan tomar con cierto grado de confianza el desafío de llevar adelante en sus aulas, prácticas matemáticas no convencionales que posibiliten la entrada de nuevas tecnologías a esas aulas.

En particular, este estudio se realizó en el marco de las primeras prácticas docentes llevadas adelante por dos futuras profesoras de matemática. Si bien las practicantes y sus acciones no estuvieron en el foco del artículo, se destaca que la posibilidad de desarrollar estas prácticas supervisadas con modelización y tecnologías contribuye a que estas dos tendencias se instalen en el horizonte de su futura actividad docente.

\section{Agradecimientos}

Agradecemos a la Dra. Cristina Esteley por haber dedicado generosamente tiempo para discutir con nosotras algunas de los conceptos teóricos que sustentan nuestro trabajo. Asimismo, agradecemos las dedicadas y agudas observaciones y sugerencias a quienes 
evaluaron el texto del artículo.

\section{Referencias}

ASINARI, M.; FRASSA, S. Experiencia de modelización matemática realizada en una escuela rural estatal con modalidad de pluricurso. In: FREGONA, D.; SMITH, S.; VILLARREAL, M; VIOLA, F. (ed.). Formación de profesores que enseñan matemática y prácticas educativas en diferentes escenarios: Aportes para la Educación Matemática. Córdoba: FAMAF-UNC, 2017. p. 161-186.

BASSANEZI, R. Temas e modelos. Campinas, Brasil: UFABC, 2012.

BOALER, J. Open and closed mathematics: student experiences and understandings. Journal for Research in Mathematics Education, Reston, v. 29, n. 1, p. 41-62, 1998.

BOALER, J. Mathematical modeling and new theories of learning. Teaching Mathematics and its Applications, Essex, v. 20, n. 3, p. 121-127, 2001.

BORBA, M.; VILLARREAL, M. Humans-with-media and the reorganization of mathematical thinking: Information and communication technologies, modelling, experimentation and visualization. New York: Springer, 2005.

BORBA, M; VILLARREAL, M.; SOARES, D. Modeling using data available on the internet. In: HIRSCH, C.; MC DUFFIE, E. (ed.). Annual Perspectives in Mathematics Education 2016: Mathematical modeling and modeling mathematics. Reston: National Council of Teacher of Mathematics, 2016. p. 143-152.

BROWN J.; IKEDA T. Conclusions and future lines of inquiry in mathematical modelling research in education. In: STILLMAN, G.; BROWN, J. (ed.). Lines of inquiry in mathematical modelling research in education: ICME-13 Monographs. Cham: Springer, 2019. p. 233-253.

CARLSON, M.; JACOBS, S.; COE, E.; LARSEN, S.; HSU, E. Applying covariational reasoning while modeling dynamic events: a framework and a study. Journal for Research in Mathematics Education, Reston, v. 33, n. 5, p. 352-378, 2002.

CARREIRA, S. Matemática e tecnologias: Ao encontro dos "nativos digitais" com os "manipulativos virtuais". Quadrante, Lisboa, v. 18, n. 1-2, p. 53-85, 2009.

CARREIRA, S.; BAIOA, A. M. Assessing the best staircase: students' modelling based on experimentation with real objects. In: CONGRESS OF THE EUROPEAN SOCIETY FOR RESEARCH IN MATHEMATICS EDUCATION - CERME, 9., 2015, Prague. Proceedings... Prague: CERME, 2015. p.834-840. Disponible en: https://hal.archives-ouvertes.fr/hal01287251/document. Acceso en: 03 dic. 2018.

CARREIRA, S.; BAIOA, A. M. Mathematical modelling with hands-on experimental tasks: on the student's sense of credibility. ZDM Mathematics Education, Karlsruhe, v. 50, n. 1-2, p. 201-215, 2018.

COIRINI, A.; GIANNONE, M. Proceso de modelización matemática. 2012.60 p. Informe final de práctica docente (Profesorado en Matemática) - Facultad de Matemática, Astronomía, Física y Computación, Universidad Nacional de Córdoba, Córdoba, 2012.

CRISTANTE, A., ESTELEY, C., MARGUET, I.; MINA, M. Experiencia de modelización en aula con orientación en Economía y Gestión de las Organizaciones. In: ABRATE, R.; POCHULU, M. (ed.). Experiencias, propuestas y reflexiones para la clase de Matemática. Villa María: UNVM, 2007. p. 
305-318.

DINIZ, L.; BORBA, M. Leitura e interpretação de dados prontos em um ambiente de modelagem e tecnologias digitais: o mosaico em movimento. Boletim de Educação Matemática, Rio Claro, v. 26, n. 43 , p. $935-62,2012$.

DOERR, H.; ÄRLEBÄCK, J.; MISFELDT, M. Representations of modelling in mathematics education. In: STILLMAN, G.; BLUM, W.; KAISER, G. (ed.). Mathematical modelling and applications: Crossing and researching boundaries in mathematics education. Cham: Springer, 2017. p. 71-82.

ESTELEY, C. Desarrollo profesional en escenarios de modelización matemática: voces y sentidos. Serie: Tesis de Posgrado. e-Book. Córdoba: FFyH, 2014. Disponible en: https://ffyh.unc.edu.ar/editorial/wp-content/uploads/sites/5/2013/05/EBOOK_ESTELEY.pdf. Acceso en: 3 dic. 2018.

GALBRAITH, P.; STILLMAN, G.; BROWN, J.; EDWARDS, I. Facilitating middle secondary modelling competencies. In: HAINES, C.; GALBRAITH, P.; BLUM, W.; KHAN, S. (ed.). Mathematical modelling: Education, engineering and economics. Chichester: Horwood, 2007. p. $130-140$.

GREEFRATH, G. Using technologies: new possibilities of teaching and learning modelling Overview. In: KAISER, G.; BLUM, W.; BORROMEO FERRI, R.; STILLMAN, G. (ed.). Trends in the teaching and learning of mathematical modeling. New York: Springer, 2011. p. 301-304.

GREEFRATH, G.; SILLER, H.-S. Modelling and simulation with the help of digital tools. In: STILLMAN, G.; BLUM, W.; KAISER, G. (ed.). Mathematical modelling and applications: Crossing and researching boundaries in mathematics education. Cham: Springer, 2017. p. 529-539.

KAISER, G. Mathematical modelling and applications in education. In: LERMAN, S. (ed.). Encyclopedia of Mathematics Education. New York: Springer, 2014. p. 396-403.

LAVE, J. La cognición en la práctica. Barcelona: Paidós, 1991.

LAVE, J. Teaching, as learning, in practice. Mind, Culture and Activity, Londres, v. 3, n. 3, p. 149164, 1996.

LAVE, J.; WENGER, E. Situated learning: Legitimate peripheral participation. Cambridge: Cambridge University Press, 1991.

MARGUET, I., ESTELEY, C., CRISTANTE, A.; MINA, M. Modelización como estrategia de enseñanza en un curso con orientación en Ciencias Naturales. In: ABRATE, R.; POCHULU, M. (ed.). Experiencias, propuestas y reflexiones para la clase de Matemática. Villa María: UNVM, 2007. p. 319-332.

MINA, M., ESTELEY, C., CRISTANTE, A.; MARGUET, I. Experiencia de modelización matemática con alumnos de 12-13 años. In: ABRATE, R.; POCHULU, M. (ed.). Experiencias, propuestas y reflexiones para la clase de Matemática. Villa María: UNVM, 2007. p. 295-304.

MINISTERIO DE EDUCACIÓN DE LA PROVINCIA DE CÓRDOBA. Diseño Curricular de la Educación Secundaria de la Provincia de Córdoba. Córdoba: ME, 2011. Disponible en: http://www.igualdadycalidadcba.gov.ar/SIPEC-

CBA/publicaciones/EducacionSecundaria/DiseniosCurricSec.php. Acceso en: 03 dic. 2018.

MOLINA-TORO, J.; RENDÓN-MESA, P.; VILLA-OCHOA, J. Research trends in digital 
technologies and modeling in mathematics education. Eurasia Journal of Mathematics, Science and Technology Education, London, v. 15, n. 8, p. 1-13, 2019.

MOLINA-TORO, J. F.; VILLA-OCHOA, J. A.; SUÁREZ TELLEZ, L. La modelación en el aula como un ambiente de experimentación-con-graficación-y-tecnología. Un estudio con funciones trigonométricas. Revista Latinoamericana de Etnomatemática, Pasto, v. 11, n. 1, p. 87-115, 2018.

MULLER, E.; BURKHARDT, H. Applications and modelling for mathematics. In: BLUM, W.; GALBRAITH, P; HENN, H.; NISS, M. (ed.). Modelling and applications in mathematics education: The 14th ICMI Study. New York: Springer, 2007. p. 267-274.

ORTEGA, M.; PUIG, L.; ALBARRACÍN, L. The influence of technology on the mathematical modelling of physical phenomena. In: STILLMAN, G.; BROWN, J. (ed.). Lines of inquiry in mathematical modelling research in education. Cham: Springer, 2019. p. 161-177.

OSBORNE, J. Teaching scientific practices: meeting the challenge of change. Journal of Science Teacher Education, London, v. 25, n. 2, p. 177-196, 2014.

REAL ACADEMIA ESPAÑOLA - RAE. Diccionario de la lengua española. 23. Ed. Disponible en: https://dle.rae.es/experimentar?m=form. Acceso en: 11 jun. 2020.

RODRÍGUEZ, R.; QUIROZ, S. El rol de la experimentación en la modelación matemática. Educación Matemática, México, v. 28, n. 3, p. 91-110, 2016.

STILLMAN, G. State of the art on modelling in mathematics education - Lines of Inquiry. In: STILLMAN, G., BROWN, J. (ed.). Lines of Inquiry in Mathematical Modelling Research in Education: ICME-13 Monographs. Cham: Springer, 2019. p. 1-20.

SWAN, M. (ed.). The language of functions and graphs. Manchester: Shell Centre Publications, 1985.

VILLARREAL, M. Modelización en la formación inicial de profesores de matemática (conferencia). In: CONFERÊNCIA NACIONAL SOBRE MODELAGEM NA EDUCAÇÃO MATEMÁTICA CNMEM, 8., 2013, Santa Maria. Anais... Santa Maria: UNIFRA, 2013. p. 1-17.

VILLARREAL, M., ESTELEY, C.; MINA, M. Modeling empowered by information and communication technologies. ZDM Mathematics Education, Karlsruhe, v. 42, n. 3-4, p. 405-419, 2010 .

VILLARREAL, M. Pensar-con-tecnologías... y educar-con-tecnologías. In: OCCELLI, M.; GARCÍA ROMANO, L.; VALEIRAS, N.; QUINTANILLA, M. (ed.). Las tecnologías de la información y la comunicación como herramientas mediadoras de los procesos educativos. V. I: Fundamentos y reflexiones. Santiago: Editorial Bellaterra Ltda, 2018. p. 57-71.

VILLARREAL, M.; ESTELEY, C. Futuros profesores de matemática: narrativas de sus primeras prácticas en escenarios de modelización. In: FREGONA, D.; SMITH, S.; VILLARREAL, M; VIOLA, F. (ed.). Formación de profesores que enseñan matemática y prácticas educativas en diferentes escenarios. Aportes para la Educación Matemática. Córdoba: FAMAF-UNC, 2017. p. 25-50.

WATSON, A. (ed.). Situated cognition and the learning of mathematics. Oxford: Centre for Mathematics Education Research/University of Oxford, 1998.

WATSON, A.; WINBOURNE, P. (ed.). New directions for situated cognition in mathematics education. New York: Springer, 2008. 
WENGER, E. Comunidades de práctica: aprendizaje, significado e identidad. Barcelona: Paidós, 2001 .

WINBOURNE, P. Looking for learning in practice: how can this inform teaching. In: WINBOURNE, P.; WATSON, A. (ed.). New directions for situated cognition in mathematics education. New York: Springer, 2008. p. 77-100.

WINBOURNE, P.; WATSON, A. Participating in learning mathematics through shared local practices in classrooms. In: WATSON, A. (ed.). Situated cognition and the learning of mathematics. Oxford: Centre for Mathematics Education Research/University of Oxford, 1998. p. 93-104.

Submetido em 12 de Dezembro de 2018. Aprovado em 18 de março de 2020. 